\title{
Feedforward and Modal Control for a Multi Degree of Freedom High Precision Machine
}

\author{
Andrea Tonoli, Angelo Bonfitto, Marcello Chiaberge, \\ Mario Silvagni, Lester D. Suarez and Enrico Zenerino
}

Additional information is available at the end of the chapter

http://dx.doi.org/10.5772/52213

\section{Introduction}

High precision industrial machines suffer the presence of vibrations mostly due to two noise sources: ground vibration and direct force disturbances. They can generate several problems at different levels and of different natures, causing performance losses on sensitive systems (Crede, 1951), (Rivin, 1979).

In the last years the growing processing quality level and the need to increase throughput resulted in a continuing demand for higher accuracy. Therefore active isolation and vibration damping systems became mandatory to satisfy these requests (Pneumont, 2002), (Hyde, 1997).

In general, machine supports are designed for high stiffness to obtain a robust machine alignment with respect to its surroundings. However, when significant ground vibration levels occur, the support stiffness is commonly sacrificed to reduce vibration transmission to the payload stage. Efforts to go towards these issues are recorded in several applications and the solutions are different for any particular situation, depending on the nature of vibration sources, the amount of disturbances and the machine environment.

Several actuation technologies are used to face this kind of problem: shape memory alloys, electromagnetic, piezoelectric, magnetostrictive and magneto-rheological fluids actuators (Thayer, 1998). Among them, electromagnetic actuators revealed themselves as effective and performing. Methods for vibration suppression can be classified in a rough approach in three families: passive, active and semi-active actuators. Completely passive solutions have almost reached their maximum potential which is still not sufficient to satisfy stringent requirements. On the opposite, the exponential growth in electronics and actuators fields made the use of active and semi-active isolation more feasible. In particular, active control 
architectures allow to perform an effective isolation at low frequencies, which is a common requirement for very demanding applications like micrometer motion control, defect inspections, critical dimensions measurement and overlay metrology.

In general, active control arrangements are provided with sensors, actuators and controllers (Watters, 1988). Each of them can be classified depending on their technology and physical working principle. The choice of sensors and actuators is strictly related to the type of application and requirements and has also influence on the selection of the control strategies to be employed. Depending on the type of controller, the system model can be used only to support the control design or can play itself a fundamental role on the control action (model based strategies) (Beadle et al, 2002), (Sullivan, 1997). Typically the main control approaches are feedback, classical or model based, and feed-forward technique, mostly with adaptive reference filtering (Anderson, 1996).

This chapter focuses on the evaluation of an active isolation and vibration damping device mounted in the working cell of a micro-mechanical laser center, which is based on active electromagnetic actuators. Two different models and three control strategies are developed and illustrated.

To clarify the goal of this study it is important to point out that: a) the vibration damping is defined as the reduction of the response amplitude of the system within a limited bandwidth near the natural frequencies of the system; b) vibration isolation is defined as the attenuation of the response of the system after its corner frequency to cut-off all the disturbances after that frequency, while allowing all the signals below it to pass with no alterations.

The machine object of study is composed by two main parts: a frame support and a payload stage where the laser cutting operation is performed. The system performance in terms of accuracy and precision is reduced by the presence of two main vibration sources: the ground and the stage itself. The active device should meet two goals: the payload vibrations damping and the reduction of the transmissibility of ground disturbances.

In this work, after a review of the major actuators families usually employed to damp and isolate high precision machines, the phases followed to design, implement and validate the proposed device are illustrated with a particular emphasis on the mechatronics aspects of the project.

A detailed analysis of the plant components is reported along with an exhaustive explanation of the design criteria followed for the choice of supports, actuation and sensing subsystems. The actuation block consists in four electromagnetic Lorentz type actuators (two per axis).

The absolute velocities of the frame support and of the stage are measured by means of eight geophone sensors to determine the amount of disturbances (Huan, 1985), (Riedesel, 1990). The considerations leading to the choice of this sensing system are reported along with the description of the related signal conditioning stage. The design of the supports between the ground and the frame and of the connections between the frame and the stage is also explained. 
Furthermore, all the subsystems described in the first part of the chapter are modeled along with their interactions. The Lagrange equations approach is used to represent the system behavior and in particular the links between the mechanical and electrical subsystems are illustrated.

Two models are developed: a) four degrees of freedom model and b) six degrees of freedom model. Both of them include the plant, the sensing, the control and the actuation blocks. Time and frequency domain computations are carried out from the models to evaluate vibration levels and displacements and to identify which control parameters need to be carefully designed to satisfy the requirements.

The last section exposes in detail the proposed control strategies along with the modeling approach validation. Three different control strategies are developed:

a. Feedback control: the control law consists in a couple of decentralized actions exerted along $X$ and $Y$-axis allowing to minimize the ground vibrations transmission and damp the payload vibrations. Specifically, a Lead-Lag control strategy, performed with a digital platform based on DSP and FPGA, is used to compensate the high-pass band dynamic of the geophone sensors and to damp the vibrations (Kuo, 1996), (Elliott, 2001). The payload isolation is achieved by feeding the control block with the difference of frame and stage velocities and giving the proper current command to the actuators. The four degrees of freedom model is used to design this control law. In the section describing the control strategy the comparisons between simulation and experimental tests is presented, which illustrates the validity of the model and the effectiveness of the proposed approach. In particular, the performance of the vibration damping has been evaluated by using the frequency responses between the actuators force and the payload velocities, whereas the performance of the active isolation is evaluated by simulating numerically the disturbances coming from the ground and evaluating their transmission through all the system till the payload in closed loop configuration.

b. Feedforward control: this action is focused on the rejection of the direct disturbance coming from the payload. The command is not generated on-line as in classical feedforward applications, but it is computed in advance from the data obtained from a direct disturbance from the payload to the machine. That is, here the compensation is computed numerically in the case of known disturbances profiles. The design of this strategy is based on the four degrees of freedom model, as in the case of the feedback control technique.

c. Modal control: the approach allows the controller to focus on the rotational and translationalal modes of the machine. Results show that the performance of this strategy are comparable to those of the standard feedback control (a), though significant advantages exist in the design procedure where the control effects can be evaluated directly on the motion modes. This technique makes use of the six degrees of freedom model.

\section{Actuators technology for damping and active isolation: An overview}

Undesired noise and vibrations are since ever a major problem in many human activities and domains. Airplanes, space trusses and satellites, cars, machine tools and large bridges, 
all can be disturbed in their normal functions by vibrations and noise. Actuators play a critical role in the active control of vibration and different technologies must be considered in order to obtain compact and efficient smart structures.

Selection and use of these technologies is greatly influenced by the user's technical knowledge, the project's budget, available energy sources, and performance tradeoffs. For example, pneumatic actuators don't deliver high force output, but are well suited when a cost-effective, easy start-up solution is required. Hydraulic actuators generate a lot of noise and can leak nasty fluid, but are ideal for high force applications that require precise control. Electromechanical actuators have high energy requirements and are more difficult to install and maintain, but are preferred for complex, multi-axis, motion control applications.

Pneumatics: pneumatic actuation is the conversion of compressed air into, typically, linear force. Typical applications involve extreme temperature and magnetic systems because pneumatic actuators don't have the magnetic field issues of electric motors. Position feedback with proximity sensors is used in modern control-loop systems, bringing pneumatics beyond simple bang-bang applications.

Pressure losses and the compressibility of air make pneumatics less efficient than other actuator technologies. In addition compressor and delivery system limitations dictate that pneumatic systems operate at lower pressures, providing lower forces and lower bandwidths than other systems. Pneumatic cylinders typically operate with compressed air at 100 psi or less, in contrast with hydraulic cylinders, which operate on pressurized hydraulic fluids at over 500 psi. Speed, force and bandwidth are directly connected with these characteristics.

Hydraulics: hydraulic actuators are suitable for rugged applications that require high force output. However, hydraulic systems generate noise and, without proper maintenance, they can leak. More equipment is needed as well: hydraulic systems require a fluid reservoir, motors and pumps, release valves, and equipment to reduce noise and heat levels. Moreover external sensors are needed to determine piston velocity, acceleration and position in a closed-loop system. Hydraulic systems can deliver much tighter control than pneumatic systems and higher force density than any other actuator technologies. Bandwidth is better than pneumatic actuators but still under hundreds of Hertz.

Electromechanical: electromechanical actuators can be based on rotatory motors (using ball screw, roller screw or belt drive), linear motors or moving coils. This type of actuator have high dynamic performance, with accelerations greater than $20 \mathrm{~g}$ and velocities of $10 \mathrm{~m} / \mathrm{sec}$ and eventually higher. Sub-micron resolution and repeatability are commonplace. Because the actuator is directly coupled to the load, there are fewer components with the chance of failure, which adds long term value.

Piezoelectric: piezomotors and piezoactuators rely on the electromechanical response of crystals. Electrical excitation causes the crystals to slightly change shape and distort, therefore generating large forces and small displacements. Exciting the crystals at a high frequency generates smooth, precise motion, making piezoelectric actuators suitable for applications with very fine positioning and high bandwidth requirements. 


\begin{tabular}{lll}
\hline $\begin{array}{l}\text { Actuator } \\
\text { Technology }\end{array}$ & Advantages & Drawbacks \\
\hline Pneumatic & Strong, light, simple, fast. & $\begin{array}{l}\text { Precise position control impossible } \\
\text { except at full stops. }\end{array}$ \\
\hline Hydraulic & Very high forces possible. & $\begin{array}{l}\text { Can leak. Requires position feedback } \\
\text { for repeatability. External hydraulic } \\
\text { pump required. Some designs good } \\
\text { in compression only. }\end{array}$ \\
\hline
\end{tabular}

\begin{tabular}{|c|c|c|}
\hline $\begin{array}{l}\text { Electro- } \\
\text { mechanical } \\
\text { rotary motor }\end{array}$ & $\begin{array}{l}\text { Cheap. Repeatable. Operation can } \\
\text { be automated. Self-contained. } \\
\text { Identical behaviour extending or } \\
\text { retracting. DC or stepping motors. } \\
\text { Position feedback possible. }\end{array}$ & Many moving parts prone to wear. \\
\hline
\end{tabular}

\begin{tabular}{lll}
\hline & Simple design. Minimum of & \\
Electro- & $\begin{array}{l}\text { moving parts. High speeds } \\
\text { mechanical }\end{array}$ & $\begin{array}{l}\text { possible. Self-contained. Identical } \\
\text { behaviour in extending or }\end{array}$ \\
& Letracting.
\end{tabular}

Force, position and speed are controllable and repeatable.
Moving coil Capable of high speeds and Requires position feedback to be precise positioning. Linear, rotary, repeatable.
and linear + rotary actions
possible.

\begin{tabular}{lll}
\hline & & $\begin{array}{l}\text { Requires position feedback to be } \\
\text { repeatable. Short travel. Low speed. }\end{array}$ \\
Piezoelectric $\quad$ Very small motions possible. & $\begin{array}{l}\text { High voltages required. Expensive. } \\
\text { Good in compression only, not in } \\
\text { tension. }\end{array}$ \\
\hline
\end{tabular}

Table 1. Actuators technology comparison

\section{System architecture}

In this section of the chapter a full description of machine subsystems is provided. The mechanical, electrical, electronic, and control parts are identified and fully described separately in the first part. Furthermore, since the project can be assumed as a classical mechatronics application, the different blocks are analyzed with their interactions in order to provide an overall view of the system. 
a)

b)
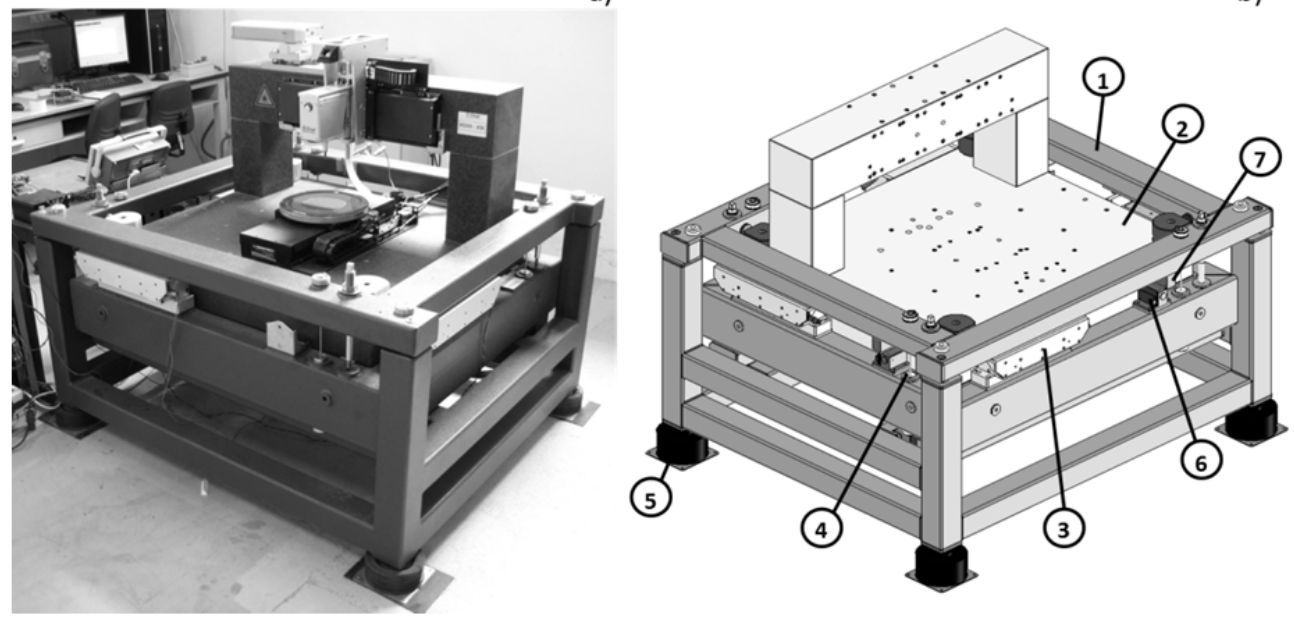

Figure 1. a) Picture of the machine. b) Sketch of the system. 1: Frame; 2: Stage; 3: Actuators; 4: FrameStage Springs; 5: Air springs; 6: Frame sensors; 7: Stage sensors.

Figure 1.a shows a picture of the laser cutting machine while in the sketch of Figure 1.b all the components of the system are highlighted. The stage (2) consists in a granitic base that can move freely within the work volume and is surrounded by four electromechanical actuators (3) acting between the frame (1) and the stage. The machine is partially isolated from the ground by means of four air springs (5). Four mechanical springs (rods) (4) are placed between the frame and the stage. The vibrations due to the machine process and coming from the ground are measured on the stage and on the frame by means of eight velocity inertial sensors $(6,7)$. A schematic representation of the actuators, sensors, and springs position is reported in Figure 2, where $\mathcal{C}_{G F}$ and $k_{G F}$ represent the damping and the stiffness, respectively introduced by the supports, whereas CFS and $k_{F S}$ are the damping and the stiffness, respectively of the springs acting as connections between frame and stage. Actuators and sensors positions can be considered collocated, in order to minimize the couplings between the axes actions by keeping the proper alternation between resonances and anti-resonances in the system dynamics. The main machine parameters and specifications are listed in Table 2. 


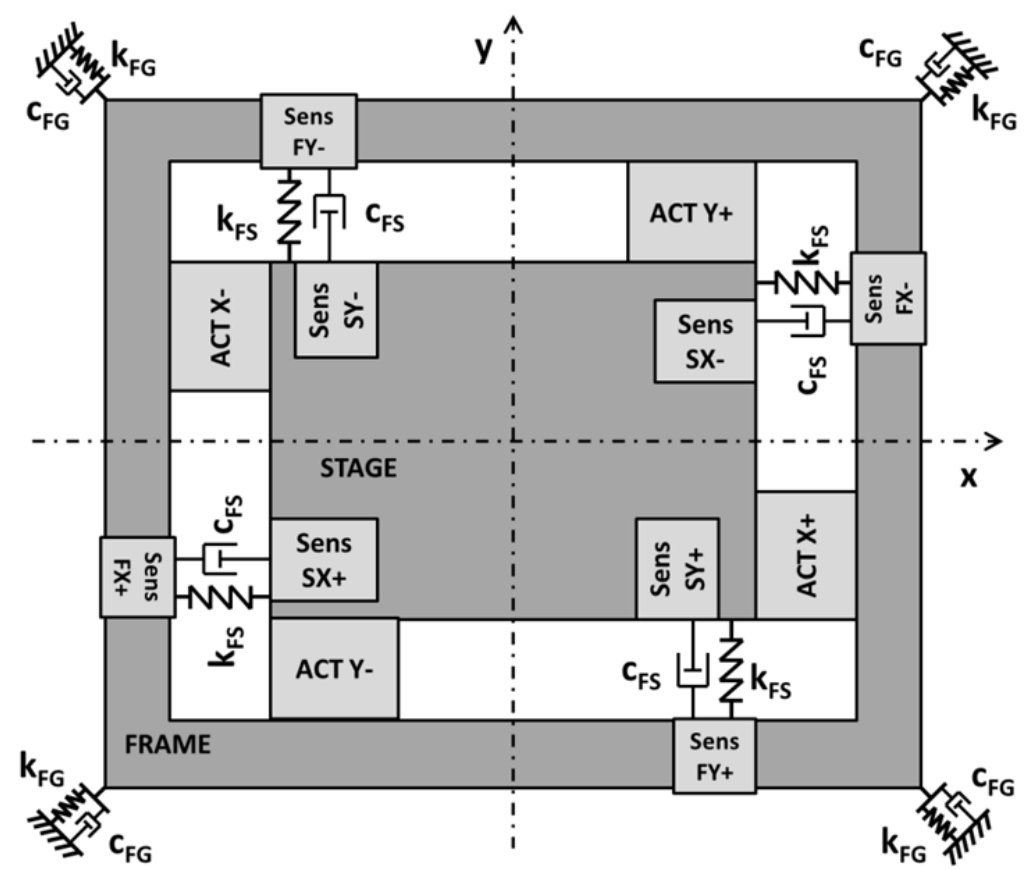

Figure 2. $X Y$ plane view of the system. Stage-Frame spring $\left(k_{S F}, c_{S F}\right)$, electromagnetic actuator (ACT), velocity sensor (Sens.), Ground-Frame spring $\left(k_{G F}, c_{G F}\right)$.

\begin{tabular}{ll}
\hline Stage mass & $1450 \mathrm{~kg}$ \\
\hline Frame mass & $300 \mathrm{~kg}$ \\
\hline Maximum displacement of the stage & $2.5 \mathrm{~mm}$ \\
\hline Inertia of the stage along $X$-axis in $Y Z$-plane & $200 \mathrm{~kg} \mathrm{~m}^{2}$ \\
\hline Inertia of the frame along $X$-axis in $Y Z$-plane & $100 \mathrm{~kg} \mathrm{~m}^{2}$ \\
\hline
\end{tabular}

Table 2. Main parameters and specifications of the machine.

The design phases have been performed considering the mechatronics nature of the system and the interactions between the machine subsystems, illustrated in Figure 3. Regarding overall controller architecture, a classical feedback behavior is performed: eight velocities are acquired by the sensors measurements and elaborate with conditioning and filtering stages in order to feed the actuators with the proper commands by means of power electronics action. The filtering stage consists in the implementation of a Lead-Lag control strategy designed to fulfill the machine requirements in terms of: a) active isolation from the disturbances coming from the ground and $b$ ) damping of the vibrations generated by the machine processes. Feedforward action is also included which allows to reject the direct disturbances coming from the payload. These feedback and feedforward control actions are completely independent one from the other. 


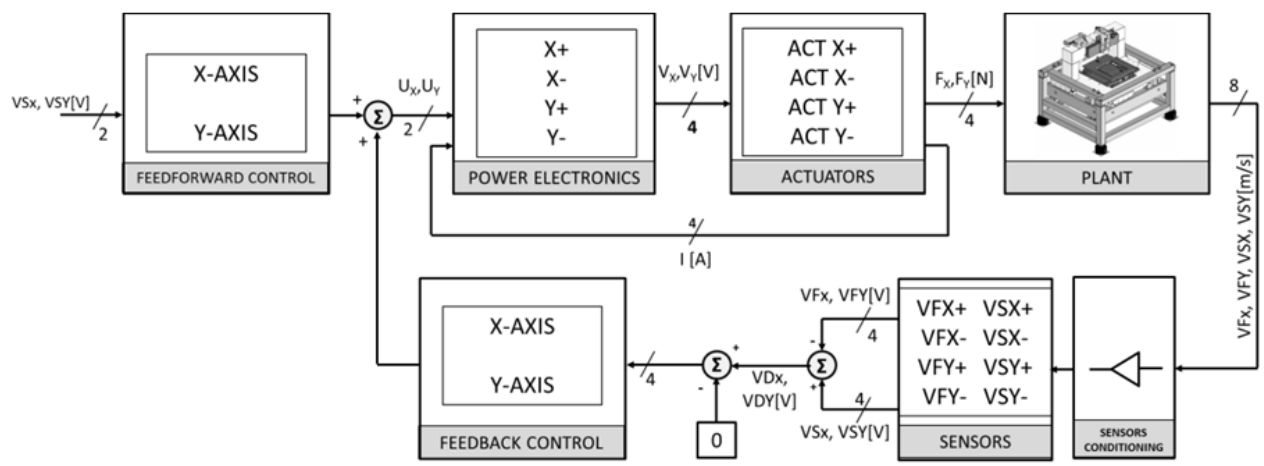

Figure 3. Block diagram of the system.

\subsection{Actuators subsystem}

The actuation on the system is realized by means of four electromagnetic Lorentz type actuators placed as illustrated in Figure 1 and Figure 2.

The picture and the section view of the actuator architecture are reported in Figure 4, being $\mathrm{A}$ and $\mathrm{B}$ permanent magnets, while $\mathrm{C}$ indicates the coil.

a)

b)
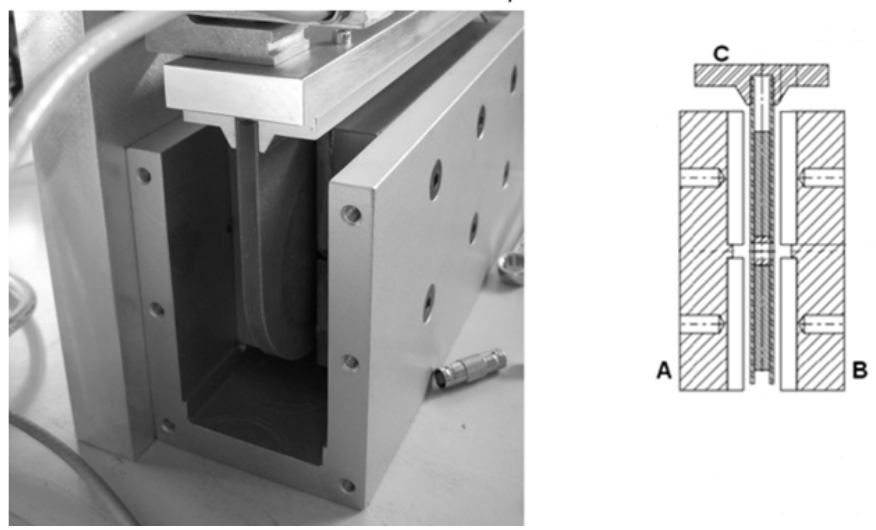

Figure 4. a) Picture of the Lorentz actuator. b) Section view (A and B: permanent magnets, C: coil).

The force $F_{A C T}$ generated by each actuator is:

$$
F_{A C T}=B N l i
$$

where $B$ is the magnetic field, $N$ is the number of turns of the coil, $i$ is the current flowing in the coil, $l$ is the coil length. The direction of the resulting force is illustrated in Figure 5. The amount of required force for each actuator is equal to $200 \mathrm{~N}$ while the main parameters of the designed actuator are reported in Table 3. 


\begin{tabular}{lll}
\hline Coil thickness & 6 & $\mathrm{~mm}$ \\
\hline Coil length & 3.3 & $\mathrm{~mm}$ \\
\hline Coil active section & 198 & $\mathrm{~mm}^{2}$ \\
\hline Copper current density & 12 & $\mathrm{~A} / \mathrm{mm}^{2}$ \\
\hline Coil length $(l)$ & 200 & $\mathrm{~mm}$ \\
\hline Coil max actuation force $\left(F_{A C T}\right)$ & 200 & $\mathrm{~N}$ \\
\hline Number of turns $(N)$ & 263 & - \\
\hline Number of coils per axis & 2 & - \\
\hline
\end{tabular}

Table 3. Actuators main parameters.

The design of the actuators has been performed starting from the requirements of force and maximum displacement of the stage, then a current density and the wire section have been selected in order to perform a FEM analysis and to compute the magnetic field. Finally, once known all the electrical parameters, the coil length $l$ has been computed.

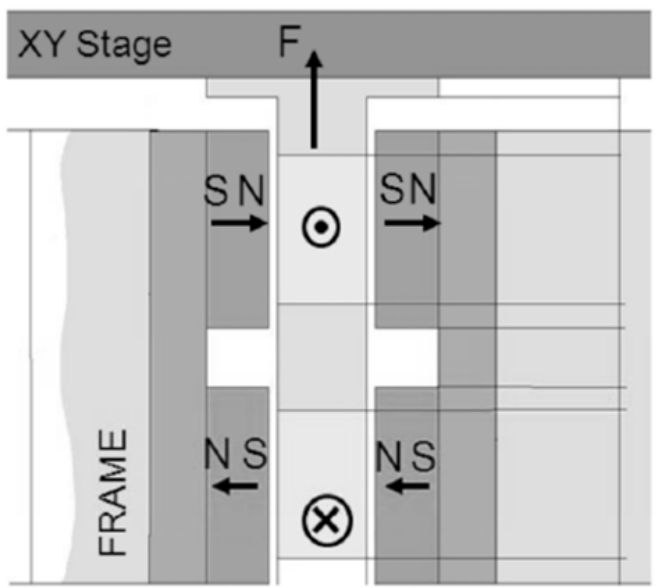

Figure 5. Actuator force generation.

The actuators parameters have been identified experimentally. The resulting values are: resistance $R=4.33 \Omega, L=9.64 \mathrm{mH}$. The actuator electrical dynamics can be expressed as:

$$
G_{A C T}(s)=\frac{1}{Z(s)}=\frac{1}{s L+R}=\frac{\frac{1}{L}}{s+\frac{R}{L}}
$$

The stationary gain $G(s=0)$ is:

$$
G(s=0)=20 \log _{10}\left(\frac{1}{R}\right)=-12.73 d B
$$


The electrical pole $\omega_{e}$ is:

$$
\omega_{e}=\frac{R}{L}=449 \mathrm{rad} / \mathrm{s}=72 \mathrm{~Hz}
$$

The resulting actuator trans-conductance (Current/Voltage) transfer function is reported in Figure 6.

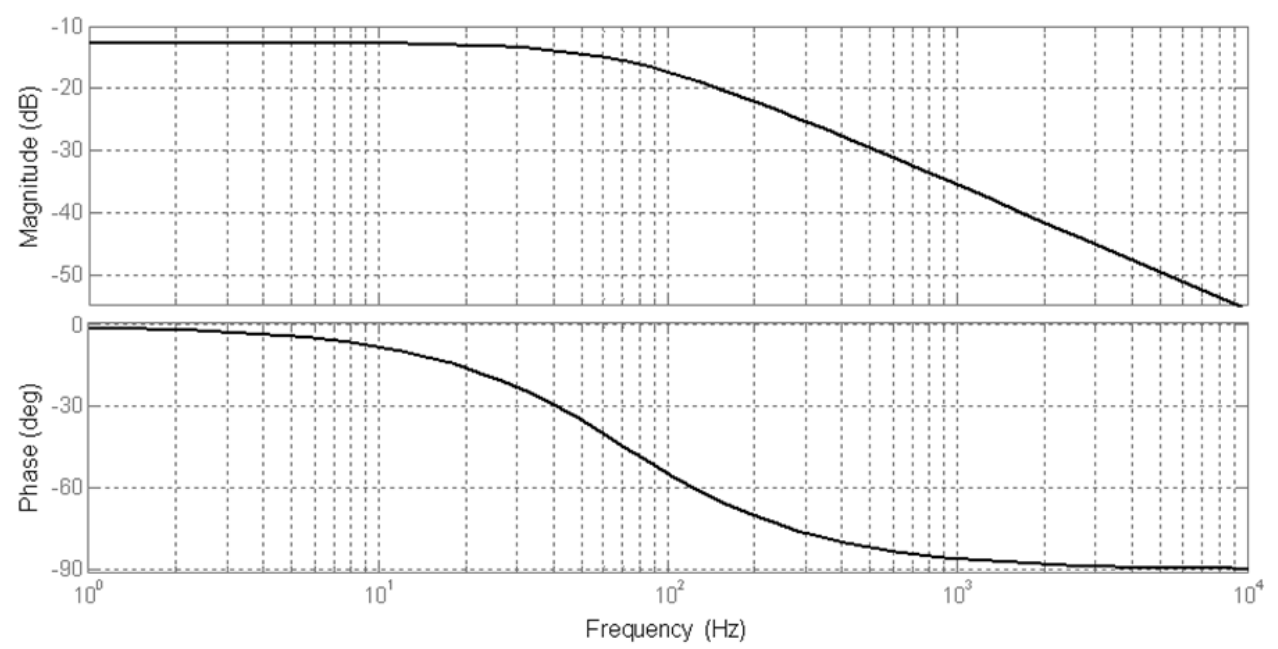

Figure 6. Actuator trans-conductance (Current/Voltage) transfer function (magnitude and phase).

\subsection{Springs and supports}

The frame and the stage are connected in the vertical direction by means of four linear springs indicated by 4 in Figure 1 as well as CSF and $k_{S F}$ in Figure 2. The design has been performed computing displacements and stresses with a FEM software, starting from the following requirements:

- infinite fatigue life;

- stiffness $\left\{\begin{array}{l}k_{S F x}=40 \mathrm{~N} / \mathrm{mm} ; \\ k_{S F y}=40 \mathrm{~N} / \mathrm{mm} ; \\ k_{S F z}=32500 \mathrm{~N} / \mathrm{mm} ;\end{array}\right.$

- damping $\left\{\begin{array}{l}c_{S F x}=228 \mathrm{Ns} / \mathrm{m} ; \\ c_{S F y}=228 \mathrm{Ns} / \mathrm{m} ; \\ c_{S F z}=4313 \mathrm{Ns} / \mathrm{m} ;\end{array}\right.$

- $\quad$ maximum displacement $z_{M A X}=2.5 \mathrm{~mm}$;

The designed spring is made of harmonic steel and is characterized by: 
- length $l_{\text {SPRING }}=125 \mathrm{~mm}$;

- $\quad$ diameter $d_{\text {SPRING }}=5 \mathrm{~mm}$;

- maximum value of stress $\sigma_{M A X}=500 M P a$.

Four air-springs (indicated by 5 in Figure 1 as well as $k_{G F}$ and $C_{G F}$ in Figure 2) consisting in a resilient element air and neoprene diaphragm, have been chosen as supports to provide the system of a partial level of isolation from the ground. The springs are characterized by the following properties:

- Nominal natural frequency: $\left\{\begin{array}{l}f_{G F x}=12.3 \mathrm{~Hz} ; \\ f_{G F y}=12.3 \mathrm{~Hz} ; \\ f_{G F z}=5.4 \mathrm{~Hz} ;\end{array}\right.$

- stiffness $\left\{\begin{array}{l}k_{G F x}=450 \mathrm{~N} / \mathrm{mm} ; \\ k_{G F y}=450 \mathrm{~N} / \mathrm{mm} ; \\ k_{G F z}=500 \mathrm{~N} / \mathrm{mm} ;\end{array}\right.$

- damping $\left\{\begin{array}{l}c_{G F x}=575 \mathrm{Ns} / \mathrm{m} ; \\ c_{G F y}=575 \mathrm{Ns} / \mathrm{m} ; \\ c_{G F z}=1700 \mathrm{Ns} / \mathrm{m} ;\end{array}\right.$

- Transmissibility at resonance: 8:1;

- The maximum load is equal to $545 \mathrm{~kg}$;

- The maximum air pressure is equal to 80 psi (5.5 bar).

\subsection{Sensing subsystem}

The disturbances on the plant are evaluated by measuring the velocities of the stage and of the frame along $X$-axis and $Y$-axis, by means of eight geophones placed as indicated in Figure 2. They are the most common inertial velocity sensors used to monitor seismic vibrations and can be classified as electromagnetic sensors that measure the velocity and produce a voltage signal thanks to the motion of a coil in a magnetic field (Hauge et al, 2002). One configuration of the conventional geophones consists of a cylindrical magnet coaxial with a cylindrical coil as shown in Figure 7. The coil is made up of a good conductor like copper and is wound around a nonconductive cylinder to avoid eddy currents effects, caused by the currents induced in the coil. The wire diameter and the dimensions of the holding cylinder are designed according to the application requirements.

The internal core is a permanent magnet selected to maximize the magnetic field density and consequently the induced voltage in the coil. The coil is fixed to the geophone housing by means of leaf springs (membranes). These springs are designed to ensure the alignment during the relative motion between coil and magnet, by keeping as low as possible the stiffness in order to minimize the geophone resonant frequency. 
The reverse configuration shown in Figure 8 is realized using a coil fixed to the housing while the moving mass is the permanent magnet. Since the mass of the magnet is heavier than that of the coil, this configuration leads to a lower natural frequency, but the moving part is larger and heavier.

a)

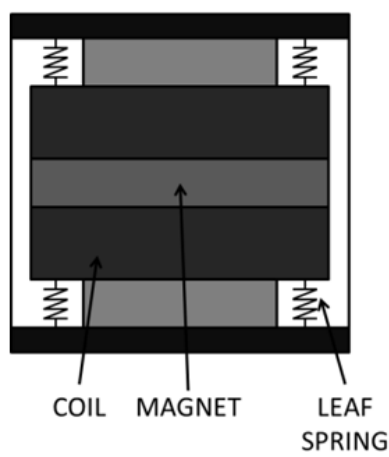

b)

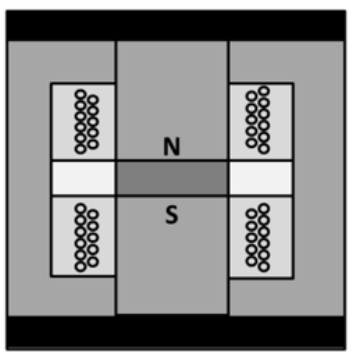

Figure 7. Geophone active configuration scheme. a) Coil and springs installation. b) Cross section.

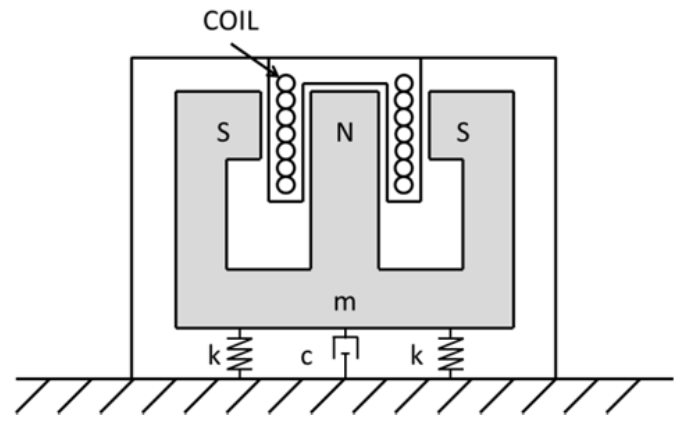

Figure 8. Geophone reverse configuration scheme.

Two different geophones of the Input-Output Inc. sensors have been tested: an active sensor model LF24 (configuration in Figure 7) and a passive sensor model SM6 (configuration in Figure 8). The LF-24 Low Frequency Geophone is characterized by the following parameters: natural frequency at $1 \mathrm{~Hz}$, distortion measurement frequency at $12 \mathrm{~Hz}$ and sensitivity equal to $15 \mathrm{~V} /(\mathrm{m} / \mathrm{s})$.

The sensor chosen is the passive model SM6 because it allows to have an extreme low noise, though the output needs to be amplified by an active conditioning stage.

The sensor response transfer between the velocity of the housing and the induced voltage in the coil, can be written in the well known second order form:

$$
T F G=-\frac{G s^{2}}{s^{2}+2 \xi \omega_{n} s+\omega_{n}^{2}}
$$


where $\omega_{n}=\sqrt{K / m}$ is the natural frequency of the geophone, $\xi=C / 2 m \omega_{n}$ is the damping ratio including the eddy current effects and $G=B l$ is the transduction constant, where $B$ is the magnetic field generated by the permanent magnet and $l$ is the length of the coil.

Considering that the first natural frequency of the system is at about $1.8 \mathrm{~Hz}$, close to the geophone natural frequency, the sensor sensitivity cannot be simply modeled as a constant value. Thus the transfer function of the geophone response must be identified to make the result more reliable.

SM6 geophone is a passive velocity sensor with the following parameters: natural frequency $4.5 \mathrm{~Hz}$ and sensitivity $28 \mathrm{~V} /(\mathrm{m} / \mathrm{s})$. The damping ratio coefficient has been experimentally identified for both sensors and is equal to 1 (model SM6 is represented in Figure 9.a and model LF24 in Figure 9.b).

Since the generated voltage is proportional to the crossing rate of the magnetic field, the output of the sensor will be proportional to the velocity of the vibrating body. A typical instrument of this kind may have a natural frequency between $1 \mathrm{~Hz}$ to $5 \mathrm{~Hz}$. The sensitivity of this kind of sensor is in the range 2-3.5 V/ms $\mathrm{m}^{-1}$ with the maximum peak to peak displacement limited to about $5 \mathrm{~mm}$ (Thomson, 1981). When a geophone is used to measure vibrations with a frequency below its natural frequency, the proof-mass tends to follow the motion of the vibrating body rather than staying stationary. This motion of the proof-mass reduces the relative motion between the same proof-mass and the housing decreasing the induced voltage. In these conditions the sensitivity of the sensor (ratio between the voltage and the casing velocity) becomes very small limiting its range of usage to frequencies above its corner frequency. It is important to underline that both displacement and acceleration can be obtained from the velocity by means integration and differentiation operations.
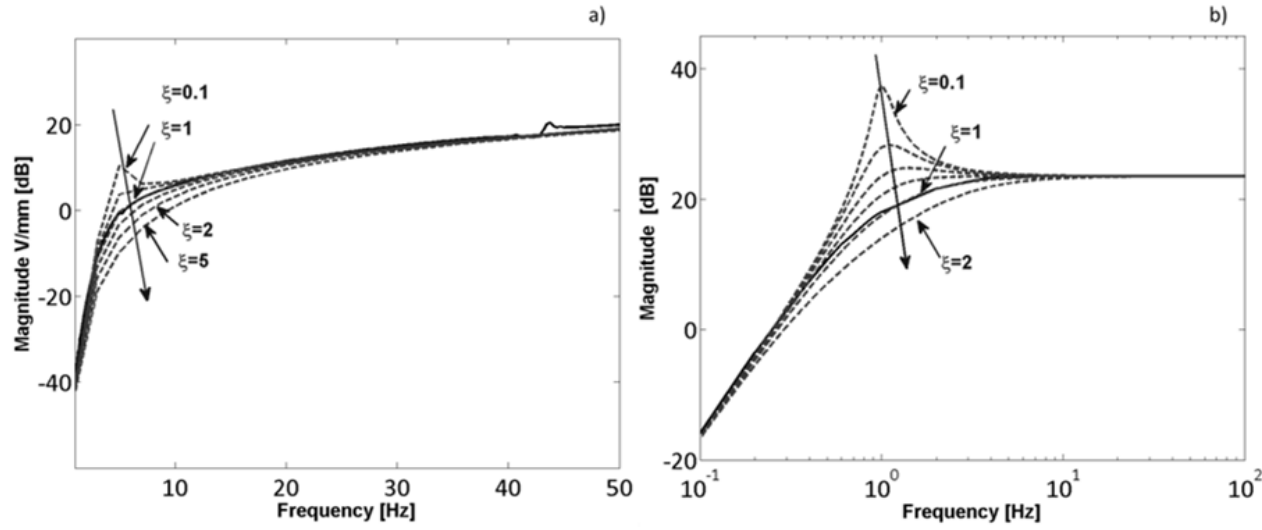

Figure 9. Geophone damping ratio identification. a) model SM6 (passive). b) model LF24 (active). 


\subsection{Electronics subsystem}

In this section the subsystems related to sensor acquisition and conditioning, power electronics and control implementation (Sensor Conditioning, Power Electronics, Feedforward Control, and Feedback Control in Figure 3) are illustrated.

The electronics system architecture is shown in Figure 10. The main characteristic of this architecture is the serial communication input/output line that provides high noise immunity, which can be useful when signals must travel through a noisy environment, such as with remote sensors.

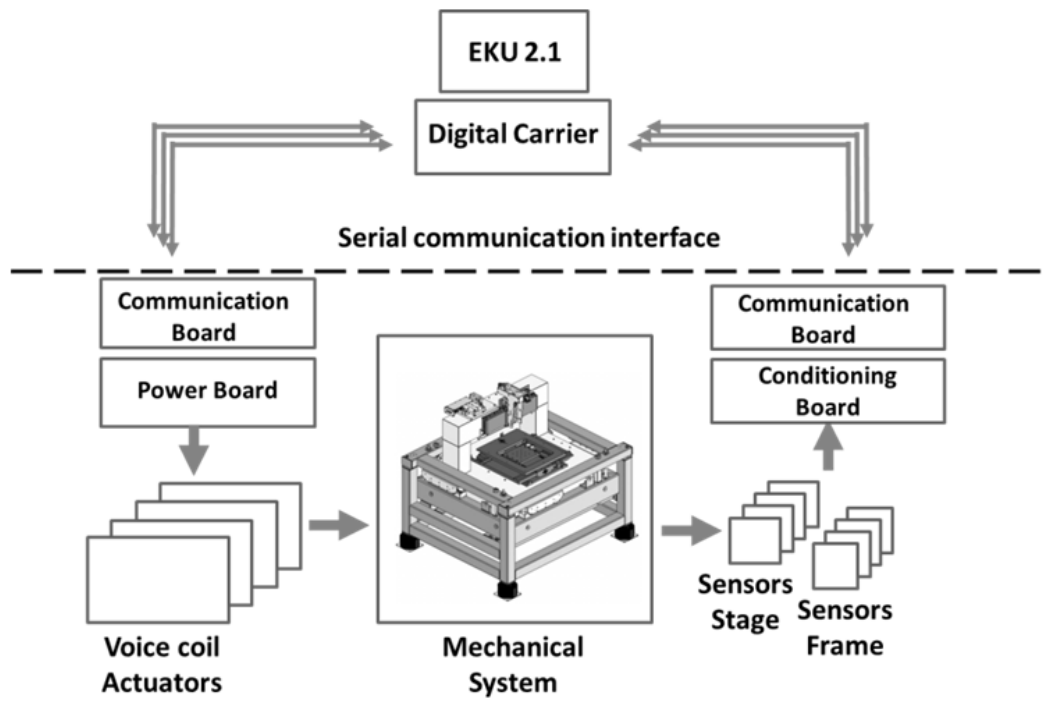

Figure 10. Electronics subsystem.

The digital carrier is used like a buffer to provide the proper current level for the serial communication. Here, multiples system buses manage data exchange between the main serial communication core (FPGA) and the communication boards placed on the plant.

The communication boards are provided with one digital-to-analog converter (DAC) and two analog-to-digital converters (ADC). The DAC is a 16-bit, high-speed, low-noise voltageoutput DAC with $30-\mathrm{MHz}$ serial interface that is capable of generating output signal frequencies up to $1 \mathrm{MHz}$. The $\mathrm{ADC}$ is a single channel 12-bit analog-to-digital converter with a high-speed serial interface and sample rate range of $50 \mathrm{ksps}$ to $200 \mathrm{ksps}$.

\section{Control Unit}

The control modules are supported by a DSP/FPGA-based digital control unit. Hence the overall control implementation can be divided between the two digital devices in order to fulfill different requirements: control strategy realization on DSP and serial communication implementation on FPGA. 
The overall control strategy is characterized with a nested and decentralized control structure, where only the outer loop is implemented on DSP while the inner current loop is realized on the power module directly. In particular, the outer loop computes the right reference for the inner one starting from required error compensation. The same strategy is applied for each axis.

\section{Sensors Conditioning}

The Sensors Conditioning Module provides the output signal from geophone by means of an instrumentation amplifier circuits. The component is configured for dual-channel operation, in order to connect two geophones together. Figure 11, shows the circuit layout for dual-channel. R1A and R1B are the gain setting resistors.

With the ADC input in the range [0-3] $\mathrm{V}$ and assuming the maximum magnitude of noise in geophone measurement nearly equal to $1000 \mathrm{~m} / \mathrm{s}$, the setting resistors are selected to achieve a gain of 100 .

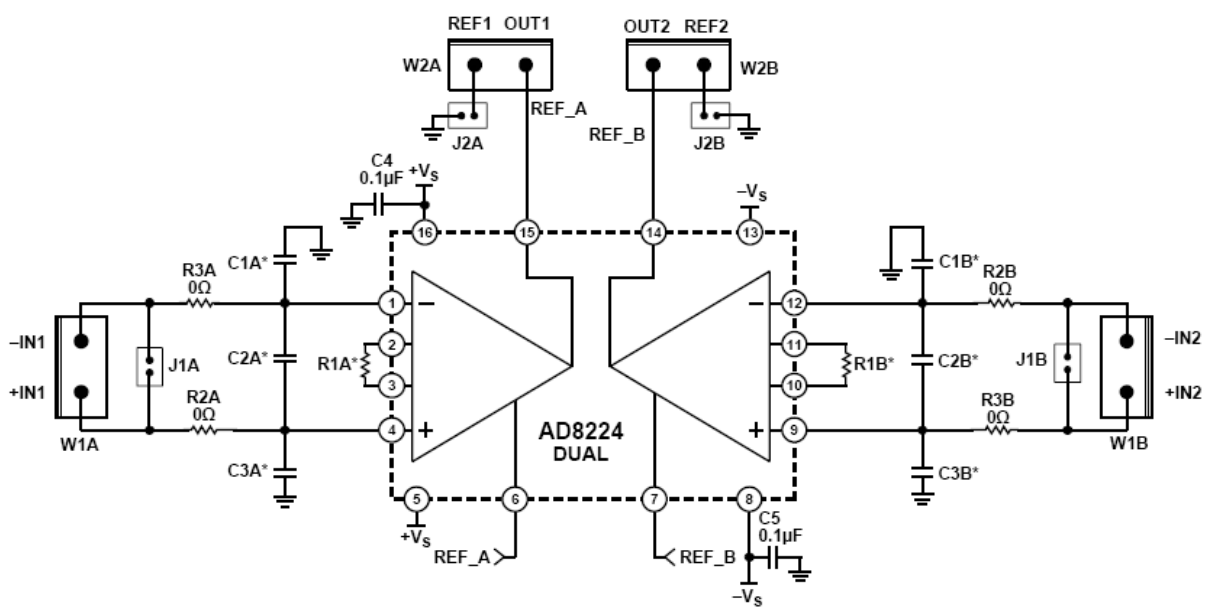

Figure 11. Instrumentation amplifier circuits AD8224. R1A and R1B are the gain setting resistors.

\section{Power Electronics}

The Power Electronics Module is based on a trans-conductance amplifier instead of a switching amplifier in order to avoid noise due to the switching frequency. This kind of amplifier operates as a voltage-to-current converter whit a differential input voltage (voltage controlled current source configuration).

The electronics layout that is divided in three main stages: a) the trans-conductance amplifier, b) the current amplifier and c) the feedback resistor.

The power module uses the voltages reference $\left(V_{i n}\right)$ from the control unit to generate the proper current $\left(I_{L}\right)$ to the load (electromagnetic actuator assumed as a RL load). The first 
stage performs the current control by means of an operational amplifier that is unity-gain stable with a bandwidth of $1.8 \mathrm{MHz}$ and it is internally protected against over-temperature conditions and current overloads. The second stage is a classical current amplifier with bipolar transistors in Darlington configuration to increase the current gain. The last stage provides the feedback signal to ensure the desired current in the load. The power supply is in the range of $\pm 30 \mathrm{~V}$.

\section{Modeling}

Two different models have been developed to permit the design of the three proposed control strategies:

1. Four degrees of freedom model used for the design of: a) a feedback controller with a Lead Lag approach, b) Feedforward control strategy.

2. Six degrees of freedom model used for the design of c) Modal controller.

\subsection{Four degrees of freedom model}

The system has been modeled by using four degrees of freedom describing the dynamics in $Y Z$ plane. Four flexural steel springs have been used to link the stage to the frame, four air springs are placed at the bottom of the frame, two actuators are working in series between the stage, and the frame and two geophones are used to measure the velocities of stage and frame respectively. As the axial stiffness of the flexural springs is very high, it can be assumed that there is no relative displacement between stage and frame along the vertical direction, which means that the relative displacement along the $\mathrm{z}$ axis between stage and frame are the same. Both stage and frame are assumed as moving about the frame mass center with the same rotating speed. The model reference frames are defined in Figure 2 (XY -plane view) and in Figure 13 (YZ -plane view).

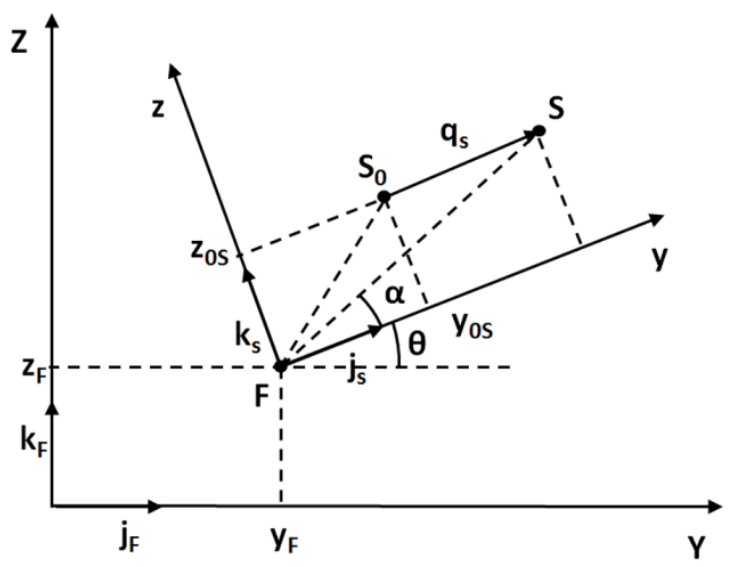

Figure 12. YZ plane 4 dof kinematic relationships scheme. 
The degrees of freedom of the model are:

$$
\bar{X}=\left[y_{F} ; z_{F} ; \theta ; \quad q_{S}\right]
$$

that indicate the displacement of the frame along $Y$-axis and $Z$-axis, the rotation of the frame (and stage) around the $X$-axis mass center and the stage displacement along its $Y$ axis.

Referring to Figure 12, it is possible to obtain the formulation of the velocity of a generic point $S$ of the stage:

$$
\begin{aligned}
& \vec{V}_{S}=\vec{V}_{F}+\dot{q}_{S} \vec{j}_{S}+\dot{\theta} \overrightarrow{F S_{0}}\left[\cos \alpha \vec{k}_{S}-\sin \alpha \vec{j}_{S}\right]= \\
& =\dot{y}_{F} \vec{j}_{F}+\dot{z}_{F} \vec{k}_{F}+\left(\dot{q}_{S}-\dot{\theta} z_{0 S}\right) \vec{j}_{S}+\dot{\theta}\left(y_{0 S}+q_{S}\right) \vec{k}_{S}
\end{aligned}
$$

The kinetic energy $T$ of the system can be expressed as:

$$
T=\frac{1}{2} m_{S} \vec{V}_{S}^{2}+\frac{1}{2} J_{S} \dot{\theta}^{2}+\frac{1}{2} m_{F} \vec{V}_{F}^{2}+\frac{1}{2} J_{F} \dot{\theta}^{2}
$$

Where $m s$ and $J s$ are the mass and the rotating inertia measured in the center of mass of the stage $S$, and $m_{F}$ and $J_{F}$ the mass and the rotating inertia measured in the center of mass of the frame $F$.

The potential energy $U$ is obtained starting from the diagram reported in Figure 13.

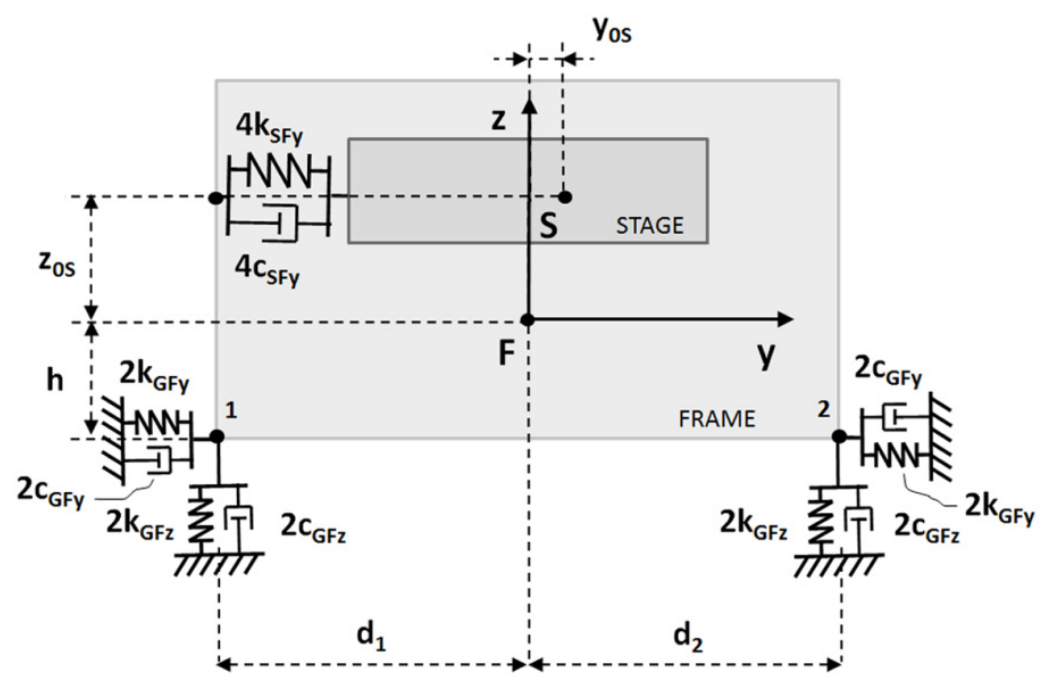

Figure 13. $Y Z$ plane 4 dof model scheme. 
The potential energy $U$ is:

$$
\begin{aligned}
& U=k_{G F z}\left(z_{F}-\theta d_{1}-z_{G}\right)^{2}+k_{G F y}\left(y_{F}+\theta h-y_{G}\right)^{2}+k_{G F z}\left(z_{F}+\theta d_{2}-z_{G}\right)^{2}+\ldots \\
& +k_{G F y}\left(y_{F}+\theta h-y_{G}\right)^{2}+k_{S F y} q_{S}^{2}
\end{aligned}
$$

where $y_{G}$ and $z_{G}$ are the displacement of the ground and $d_{1}, d_{2}$, and $h$ the quantities reported in Figure 13.

Owing to the Rayleigh formulation, the damping of the system is governed by the following dissipation function:

$$
\begin{aligned}
& \Re=c_{G F z}\left(\dot{z}_{F}-\dot{\theta} d_{1}-\dot{z}_{G}\right)^{2}+c_{G F y}\left(\dot{y}_{F}+\dot{\theta} h-\dot{y}_{G}\right)^{2}+c_{G F z}\left(\dot{z}_{F}+\dot{\theta} d_{2}-\dot{z}_{G}\right)^{2}+\ldots \\
& +c_{G F y}\left(\dot{y}_{F}+\dot{\theta} h-\dot{y}_{G}\right)^{2}+c_{S F y} \dot{q}_{S}^{2}
\end{aligned}
$$

where each damping term $c_{i}$ is obtained starting from the experimental identification of damping ratios $\varsigma_{i}$ :

$$
c_{i}=2 \varsigma_{i} \sqrt{k_{i} m_{i}}
$$

The inputs of the system are: the force of the electromagnetic actuators $F_{a c t}$, the force of the stage $F_{S}$ and the velocities from the ground in y direction $v_{G y}$ and $z$ direction $v_{G z}$. The output are the velocities $v_{F}$ of the frame and $v_{S}$ of the stage measured with geophones sensors. Inputs and outputs are graphically represented in Figure 14.

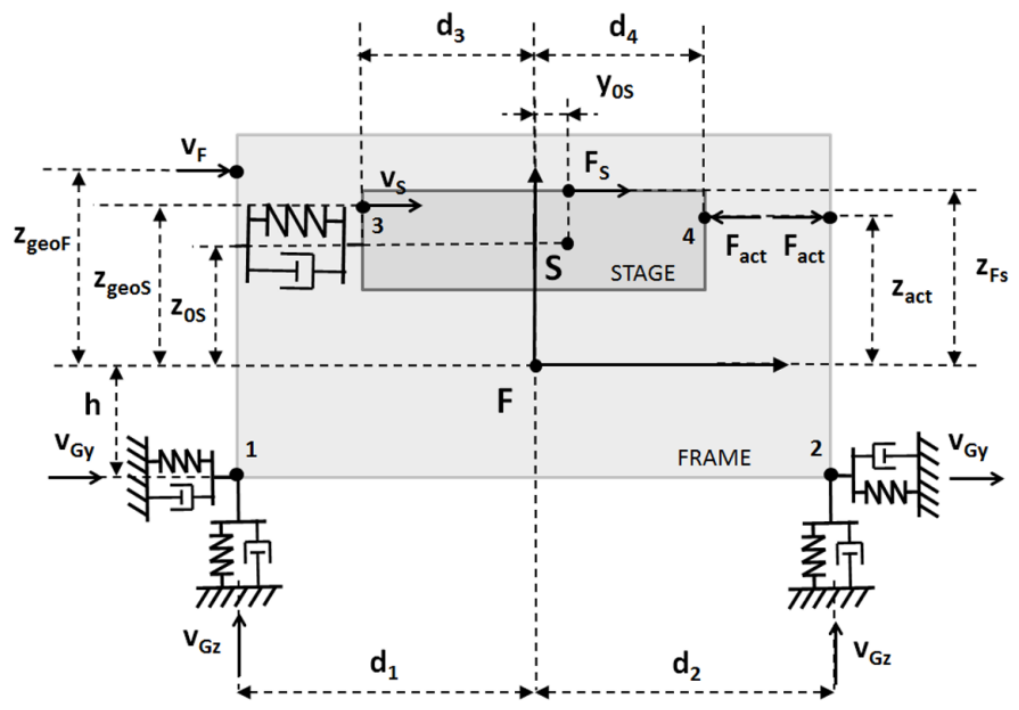

Figure 14. YZ plane 4dof model scheme - input and output. 
Using the Lagrange formulation is possible to write the equations of motion in the form:

$$
\mathrm{M} \ddot{q}+C \dot{q}+\mathrm{K} q+T_{G} q_{G}=\mathrm{T}\{F\}
$$

where

$$
\begin{gathered}
q=\left(\begin{array}{cccc}
y_{F} & z_{F} & \vartheta & q_{S}
\end{array}\right)^{T} \\
q_{G}=\left(\begin{array}{ll}
y_{G} & z_{G}
\end{array}\right)^{T}
\end{gathered}
$$

are the vectors of the generalized coordinates,

$$
F=\left(\begin{array}{llll}
v_{G y} & v_{G z} & F_{S} & F_{a c t}
\end{array}\right)^{T}
$$

is the vector of the generalized forces and $\mathrm{M}$ is the mass matrix

$$
M=\left[\begin{array}{cccc}
m_{t o t} & 0 & -m_{S} z_{0 S} & m_{S} \\
0 & m_{t o t} & m_{S} y_{0 S} & 0 \\
-m_{S} z_{0 S} & m_{S} y_{0 S} & J_{t o t} & -m_{S} z_{0 S} \\
m_{S} & 0 & -m_{S} z_{0 S} & m_{S}
\end{array}\right]
$$

with $m_{\text {tot }}=m_{S}+m_{F}, J_{t o t}=J_{S}+J_{F}+m_{S}\left(y_{0 S}{ }^{2}+z_{0 S}{ }^{2}\right) . y_{0 S}, z_{0 S}$ are the initial position of the stage. The matrix is symmetric and not diagonal because it takes into account the coupling between the stage and the frame dynamics.

The stiffness matrix $K$ is:

$$
K=\left[\begin{array}{cccc}
4 k_{G F y} & 0 & 4 k_{G F y} h & 0 \\
0 & 4 k_{G F z} & -2 k_{G F z} d_{1}+2 k_{G F z} d_{2} & 0 \\
4 k_{G F y} h & -2 k_{G F z} d_{1}+2 k_{G F z} d_{2} & 2 k_{G F z} d_{1}^{2}+4 k_{G F y} h^{2}+2 k_{G F z} d_{2}^{2} & 0 \\
0 & 0 & 0 & 4 k_{S F y}
\end{array}\right]
$$

The damping matrix $C$ is:

$$
C=\left[\begin{array}{cccc}
4 c_{G F y} & 0 & 4 c_{G F y} h & 0 \\
0 & 4 c_{G F z} & -2 c_{G F z} d_{1}+2 c_{G F z} d_{2} & 0 \\
4 c_{G F y} h & -2 c_{G F z} d_{1}+2 c_{G F z} d_{2} & 2 c_{G F z} d_{1}^{2}+4 c_{G F y} h^{2}+2 c_{G F z} d_{2}^{2} & 0 \\
0 & 0 & 0 & 4 c_{S F y}
\end{array}\right]
$$

The selection matrix $\mathrm{T}$ of the generalized forces is: 


$$
\begin{gathered}
T=\left[\begin{array}{cccc}
-4 c_{G F y} & 0 & 1 & 0 \\
0 & -4 c_{G F z} & 0 & 0 \\
4 c_{G F y} h & 2 c_{G F z} d_{1}-2 c_{G F z} d_{2} & 0 & 0 \\
0 & 0 & 1 & -1
\end{array}\right] \\
T_{G}=\left[\begin{array}{cc}
-4 k_{G F y} & 0 \\
0 & 4 k_{G z y} \\
4 k_{G F y} h & 2 k_{G F z} d_{1}-2 k_{G F z} d_{2} \\
0 & 0
\end{array}\right]
\end{gathered}
$$

In the state space formulation the equations of motion of the system can rewritten as:

$$
\dot{X}=A X+B U
$$

where the state vector $X$ and the input vector are:

$$
X=\left\{\begin{array}{ccc}
q & \dot{q} & q_{G}
\end{array}\right\}^{T}, U=\left\{\begin{array}{llll}
v_{G y} & v_{G z} & F_{S} & F_{a c t}
\end{array}\right\}^{T}
$$

with A the state matrix, $\mathrm{B}$ the input matrix

$$
A=\left[\begin{array}{ccc}
0 & I & 0 \\
-M^{-1} K & -M^{-1} C & M^{-1} T_{G} \\
0 & 0 & 0
\end{array}\right], \quad B=\left[\begin{array}{c}
0 \\
M^{-1} T \\
I
\end{array}\right]
$$

The relationship between input and output can be represented as:

$$
Y=C X+D U
$$

where $Y$ is the output vector, $C$ the output matrix and $D$ the feedthrough matrix

$$
Y=\left\{\begin{array}{ll}
v_{S} & v_{F}
\end{array}\right\}^{T}, C=\left[\begin{array}{llllllllll}
0 & 0 & 0 & 0 & 1 & 0 & -z_{\text {geoS }} & 1 & 0 & 0 \\
0 & 0 & 0 & 0 & 1 & 0 & -z_{g e o F} & 1 & 0 & 0
\end{array}\right], D=\left[\begin{array}{llll}
0 & 0 & 0 & 0 \\
0 & 0 & 0 & 0
\end{array}\right]
$$

\subsection{Six degrees of freedom model}

As well as the dynamics on the $\mathrm{YZ}$ plane described in the previous section, it has been developed a six degrees of freedom model of system dynamics on the XY plane. In this case, the degrees of freedom of the model are:

$$
\bar{X}=\left[x_{S} ; y_{S} ; \theta_{S} ; x_{F} ; y_{F} ; \theta_{F}\right]
$$


indicating the stage displacements $x_{s}$ along $\mathrm{X}$-axis, $y s$ along $\mathrm{Y}$-axis, the rotation $\theta$ s about the axis passing through the mass center and oriented along the Z-axis, the frame displacements $x_{F}$ along $\mathrm{X}$-axis, $y_{F}$ along $\mathrm{Y}$-axis, and the rotation $\theta_{\mathrm{F}}$ about the axis passing through the mass center oriented along the Z-axis. Stage and frame degrees of freedom, inputs, and geometric properties are illustrated in Figure 15 and 16.

Resorting to the Lagrange formulation as reported in (12), the $q$ vector of the generalized coordinates is:

$$
q=\left(\begin{array}{llllll}
x_{S} & y_{S} & \theta_{S} & x_{F} & y_{F} & \theta_{F}
\end{array}\right)^{T}
$$

and the $F$ the vector of the generalized forces is

$$
F=\left(\begin{array}{llll}
F_{X+} & F_{X-} & F_{Y_{+}} & F_{Y_{-}}
\end{array}\right)^{T}
$$

it is possible to obtain the corresponding mass matrix $\mathrm{M}$, stiffness matrix $\mathrm{K}$ and damping matrix C (not reported due to its excessive size).

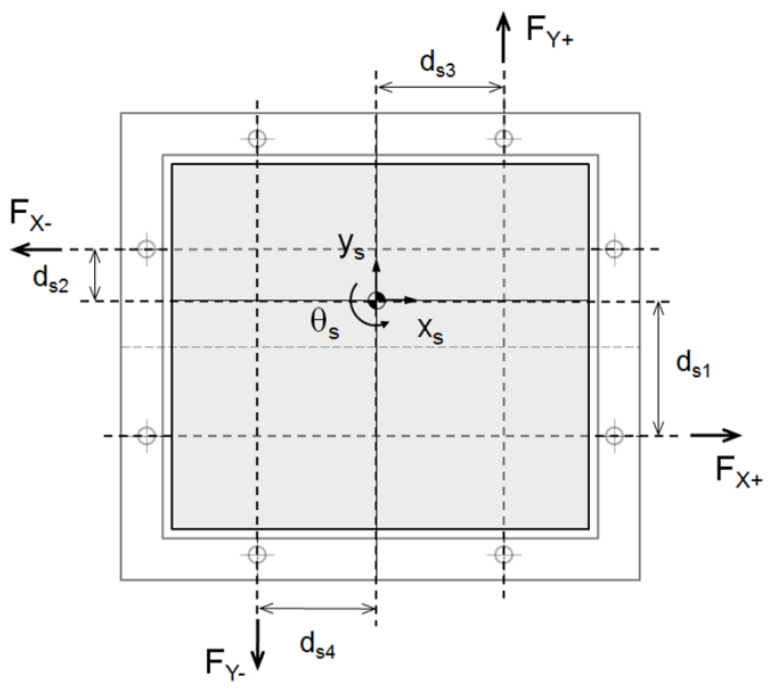

Figure 15. XY Plane 6 dof model scheme: stage degrees of freedom and inputs. 


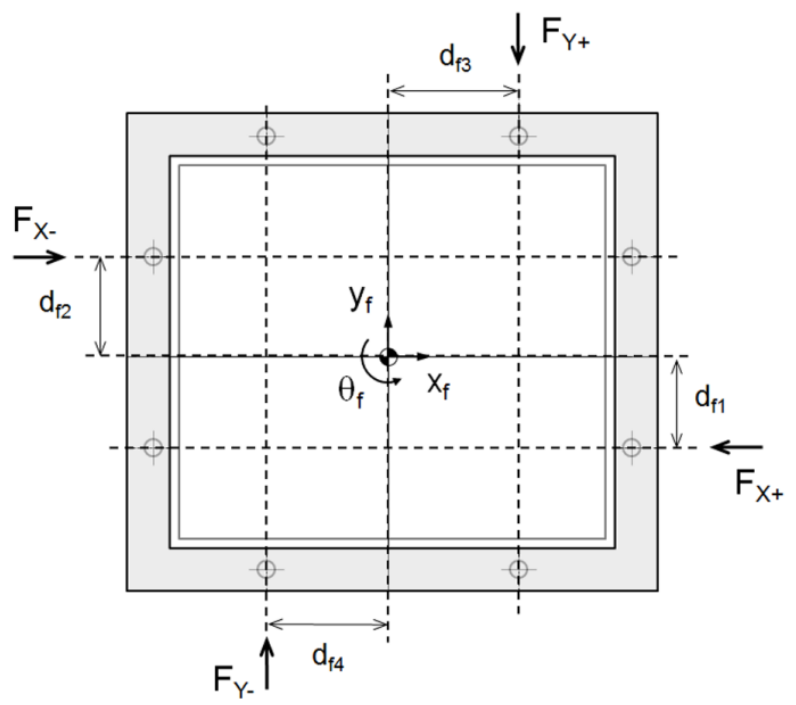

Figure 16. XY Plane 6 dof model scheme: stage degrees of freedom and inputs.

The selection matrix $\mathrm{T}$ of the generalized forces is:

$$
T=\left[\begin{array}{cccc}
1 & -1 & 0 & 0 \\
0 & 0 & 1 & -1 \\
d_{s 1} & d_{s 2} & d_{s 3} & d_{s 4} \\
-1 & 1 & 0 & 0 \\
0 & 0 & -1 & 1 \\
-d_{f 1} & -d_{f 2} & -d_{f 3} & -d_{f 4}
\end{array}\right]
$$

Similarly in the state space formulation the equations of motion of the system can rewritten as:

$$
\dot{X}=A X+B U
$$

where the state vector $\mathrm{X}$ and the input vector $U$ are:

$$
X=\left\{\begin{array}{ll}
q & \dot{q}
\end{array}\right\}^{T}, U=\{F\}^{T}
$$


with the following state and input matrix

$$
A=\left[\begin{array}{cc}
0 & I \\
-M^{-1} K & -M^{-1} C
\end{array}\right], B=\left[\begin{array}{c}
0 \\
M^{-1} T
\end{array}\right]
$$

The relationship between input and output can be represented as:

$$
Y=C X+D U
$$

where $Y$ is the output vector that contains the derivative time of the generalized coordinates (25):

$$
Y=\{\dot{q}\}^{T}
$$

C is the output matrix and $D$ the feedthrough matrix:

$$
C=\left[\begin{array}{ll}
0 & I
\end{array}\right], D=[0]
$$

\section{Control design \& results}

In this section three different control strategies to damp vibration and isolate the machine are proposed: a) Feedback control by the use of a Lead-Lag technique, b) Feedforward control and c) Modal control. The experimental validation has been carried out just for the first strategy as proof of the correctness of the modeling approach. Feedforward and modal controls are validated numerically.

\subsection{Feedback control}

The control action is designed to achieve two main goals: active isolation of the payload from the ground disturbances and vibration damping during the machine work processes. These two actions allow to operate on the stage without external disturbances. Dynamics on $X Z$ and $Y Z$-planes are considered the same and decoupled so the control laws along the two planes are equivalent.

Furthermore, from the control point of view, the adopted model is oversized with respect to the control requirements if the goal is the isolation of the stage. As a matter of fact, in this case a two degrees of freedom model is sufficient while if also the dynamics of the frame is required to be controlled, then a 4 dof model is necessary.

The considered system can be regarded as intrinsically stable due to the presence of mechanical stiffness between the stage and the frame, which allows to obtain a negative real part for all the eigenvalues of the system.

Root loci of the system in open and closed loop configurations are reported in Figure 17. 

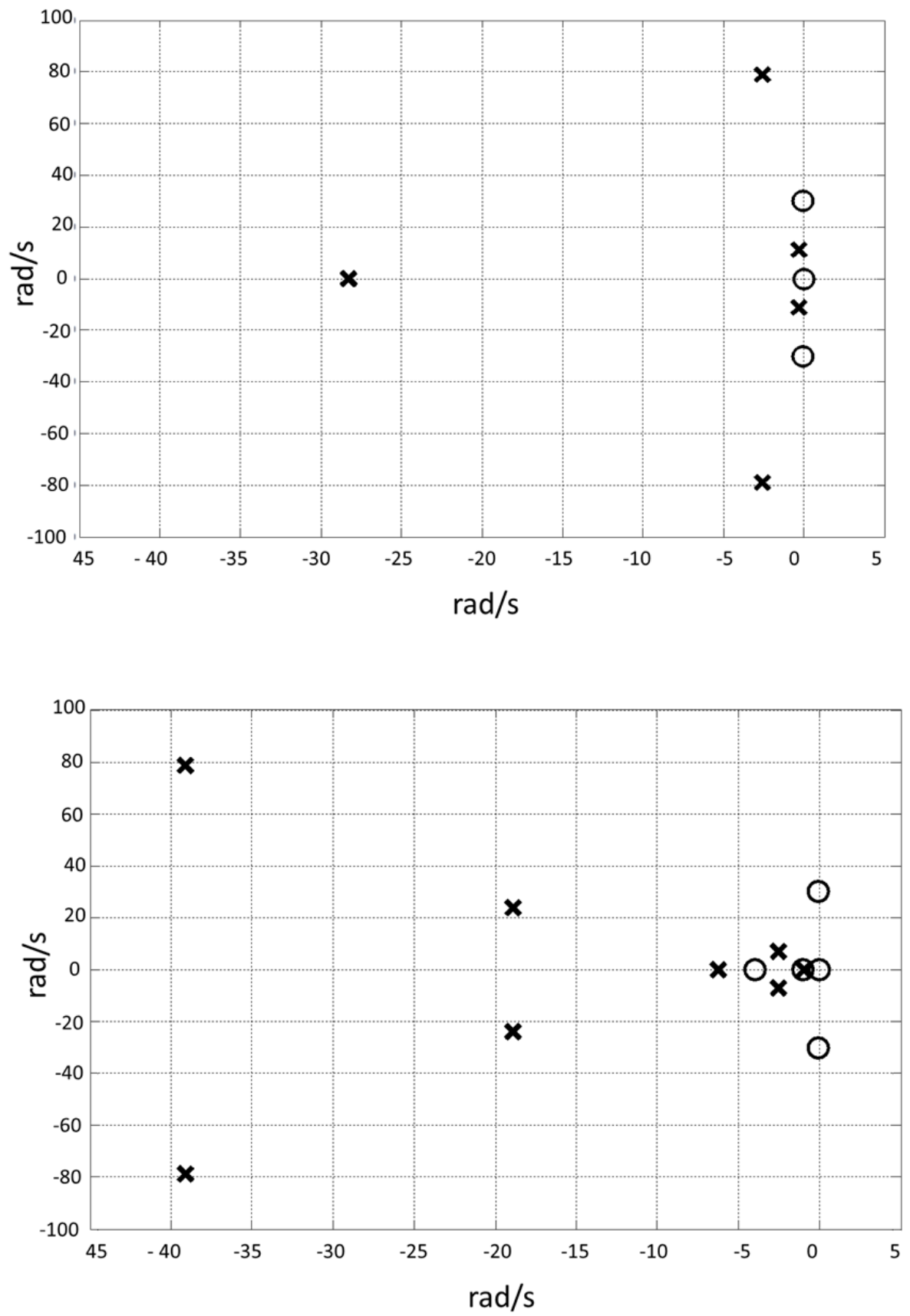

Figure 17. Root loci of open loop (a) and closed loop (b) configurations (Circles: zeros; Crosses: poles). 
Poles and zeros of the system are reported in Table 4.

\begin{tabular}{|c|c|c|}
\hline & Poles [rad/s] & Zeros [rad/s] \\
\hline \multirow{2}{*}{ 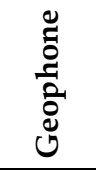 } & -28.2743 & - \\
\hline & -28.2743 & - \\
\hline \multirow{2}{*}{ 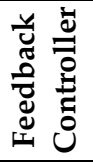 } & -1 & -75 \\
\hline & -4 & -12 \\
\hline \multirow{10}{*}{ 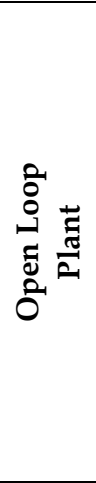 } & $-6.4136+79.8805 i$ & $-5.8786+80.8581 i$ \\
\hline & $-6.4136-79.8805 i$ & $-5.8786-80.8581 i$ \\
\hline & $-3.7383+46.4898 \mathrm{i}$ & $-2.0791+37.9468 \mathrm{i}$ \\
\hline & $-3.7383-46.4898 \mathrm{i}$ & $-2.0791-37.9468 \mathrm{i}$ \\
\hline & $-0.2371+10.9133 i$ & $-1.9325+33.6912 i$ \\
\hline & $-0.2371-10.9133 \mathrm{i}$ & $-1.9325-33.6912 i$ \\
\hline & $-1.9557+33.7951 \mathrm{i}$ & 0 \\
\hline & $-1.9557-33.7951 \mathrm{i}$ & 0 \\
\hline & -28.2743 & 0 \\
\hline & -28.2743 & - \\
\hline \multirow{12}{*}{ 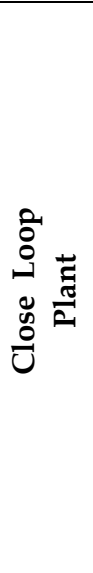 } & $-6.3137+79.8109 \mathrm{i}$ & $-5.8786+80.8581 i$ \\
\hline & $-6.3137-79.8109 i$ & $-5.8786-80.8581 \mathrm{i}$ \\
\hline & $-5.3540+46.2140 \mathrm{i}$ & $-2.0791+37.9468 \mathrm{i}$ \\
\hline & $-5.3540-46.2140 \mathrm{i}$ & $-2.0791-37.9468 \mathrm{i}$ \\
\hline & $-1.9649+33.7933 \mathrm{i}$ & $-1.9325+33.6912 \mathrm{i}$ \\
\hline & $-1.9649-33.7933 \mathrm{i}$ & $-1.9325-33.6912 \mathrm{i}$ \\
\hline & $-27.7026+12.6862 i$ & -1 \\
\hline & $-27.7026-12.6862 \mathrm{i}$ & -4 \\
\hline & $-1.8090+9.2149 \mathrm{i}$ & 0 \\
\hline & $-1.8090-9.2149 i$ & 0 \\
\hline & -4.7413 & 0 \\
\hline & -0.9885 & - \\
\hline
\end{tabular}

Table 4. Poles and zeros of the system

Since the system along $Y Z(X Z)$ presents one actuation point and a couple of sensors (frame and stage velocities), a solution with a SISO control strategy is not feasible. A simplest solution to this problem considers the difference between the measured velocities as the feedback signal, so the system can be assumed as SISO and the control design becomes simpler. 
Figure 18 shows that the system dynamics has a peak at $1.8 \mathrm{~Hz}$ related to the stage and higher modes related to the interaction of the stage with the frame and the ground at $10 \mathrm{~Hz}$ and beyond.

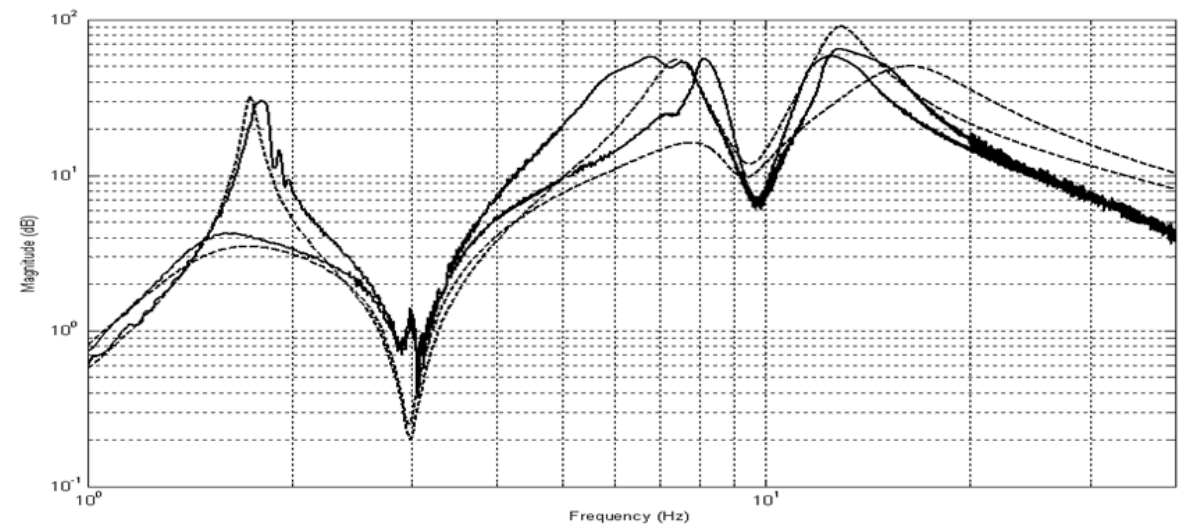

Figure 18. Vibration damping action. Transfer function from the actuator force to the difference of frame and stage velocities $\left(\left(\dot{q}_{S}-\dot{q}_{F}\right) / F_{A C T}\right)$. Open-loop vs Closed-loop. Solid line: experimental; Dashed line: numerical.

The feedback controller is focused on damping the mode related to the stage by adding on the loop a lead-lag compensator.

The two actions can be expressed as:

$$
\begin{aligned}
& C_{\text {LAG }}=\frac{s+z_{L A G}}{s+p_{L A G}} \\
& C_{L E A D}=\frac{s+z_{L E A D}}{s+p_{L E A D}}
\end{aligned}
$$

The $C_{L A G}$ action is used to improve the transient response at low frequency, while the $C_{L E A D}$ is useful to increase the stability margin of the closed-loop system.

Therefore the resulting Lag-Lead action allows to compensate the critical phase behavior of the geophones and furthermore guarantees a quick damping action with good levels of stability margins.

The experimental tests have been performed to validate the two control actions. Figure 18 shows the numerical and experimnental frequency response function in open loop and closed loop, obtained from the actuator force to the velocity measured on the stage. The force acts both on the stage and the frame, the dynamics of both the subsystems are visible. The vibration damping effect of the control action is validated on the stage mode $(1.8 \mathrm{~Hz}$ peak) and the good correspondence shown between the simulated and experimental response is useful to validate the modeling approach. 

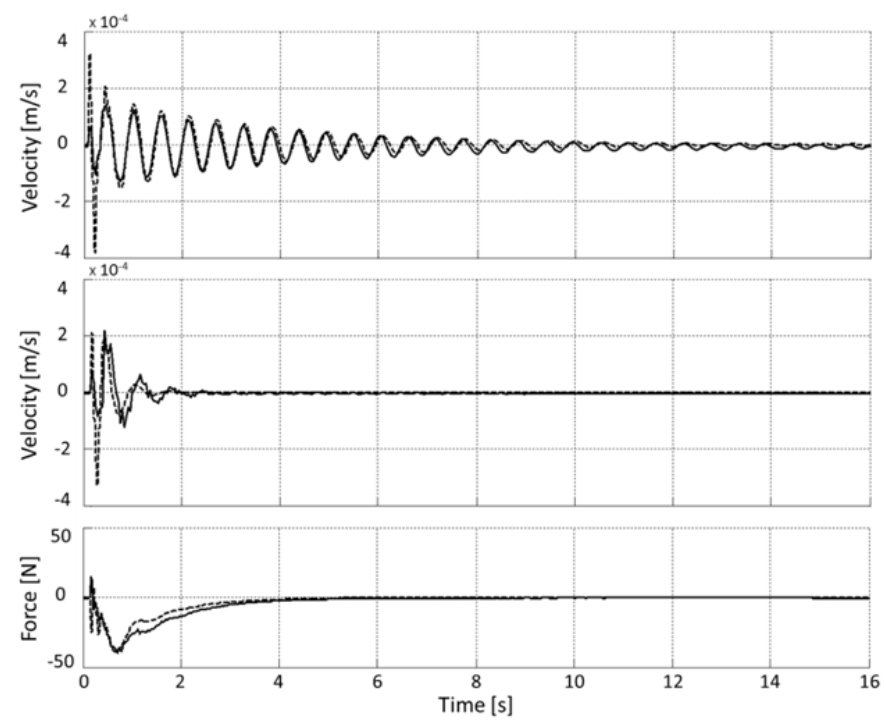

Figure 19. Impulse time response, force from the actuator and velocity measured on the stage. Openloop (a), Closed-loop (b), Force exerted by the actuators. Solid line: experimental results. Dashed line: numerical results.

A further demonstration of the correctness of the damping action is the velocity time response reported in Figure 19. In this case the system is excited with an impulse from the actuator and the velocity is measured on the stage. Numerical and experimental responses are superimposed to provide a further validation of the model (the position time response is not reported since the machine is not provided with displacement sensors and hence this validation could not be possible to performed). Figure 19.a shows open loop response, Figure 19.b shows closed loop response while in Figure 19.c the force exerted by the actuators is reported.

The excitation coming from the laser-axis action on the stage is controlled in an effective way as shown in Figure 20 where the numerical transfer function between a force impulse on the stage and the related measured velocity is reported. 


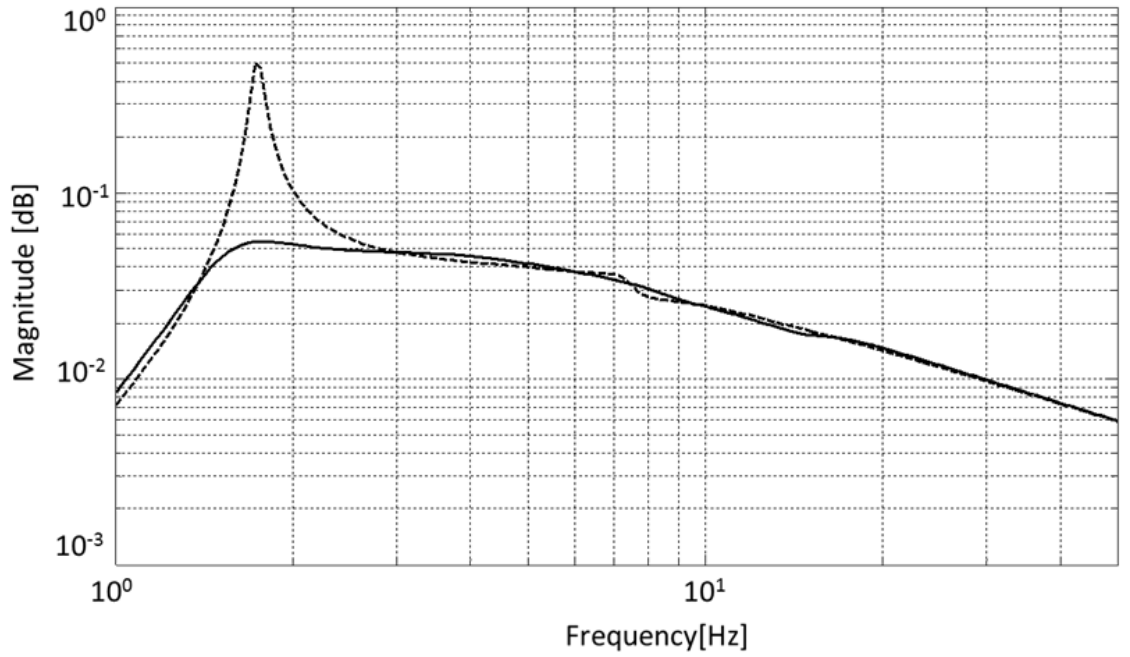

Figure 20. Vibration damping action. Transfer function from a force applied on the stage to the velocity measured on the stage $\left(\dot{q}_{S} / F_{S}\right)$. Numerical response. Solid line: closed-loop; Dashed line: Open-loop.

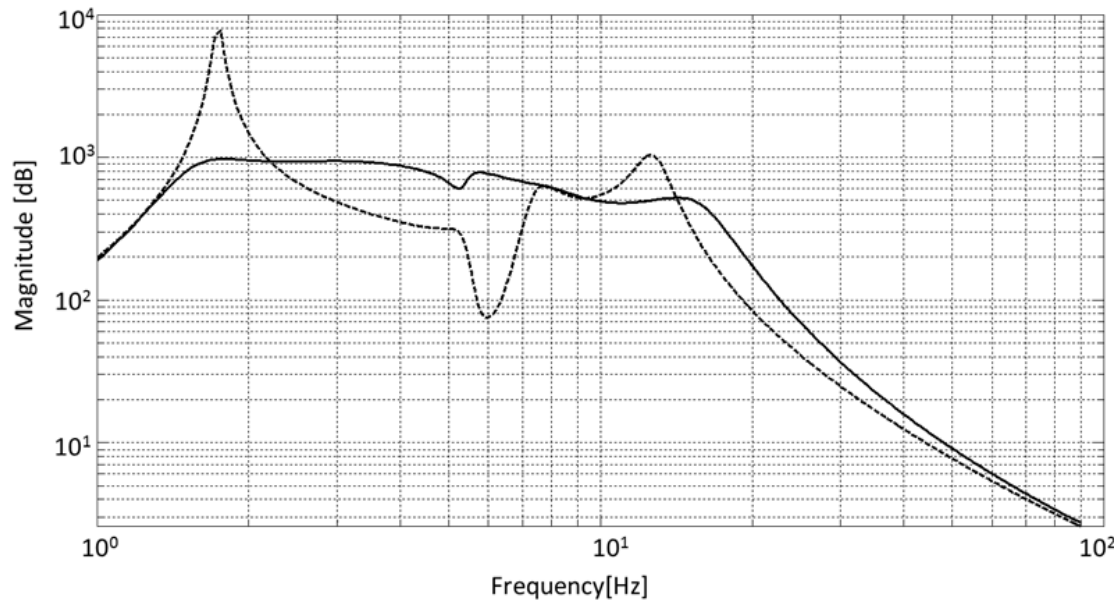

Figure 21. Active isolation action. Transfer function from a simulated ground velocity to the velocity measured on the stage $\left(\dot{q}_{S} / \dot{q}_{G}\right)$. Numerical response. Solid line: Open loop configuration. Dashed line: Closed loop configuration.

The active isolation action is verified by simulating the excitation coming from the ground. The experimental test in this case has not been performed since in reality it is difficult to excite the machine from the ground in a controlled and effective way. Nevertheless the model is reliable as proved in Figure 14 and the obtained results can be assumed as a good validation of the control action. 
Figure 21 illustrates that the closed loop system is capable to reject the disturbances coming from the ground in an effective way.

\subsection{Feedforward control}

Although the feedback control explained in Section 5.1 is strongly effective for external disturbances coming from the ground, it could not be sufficient to make the machine completely isolated from the direct disturbance generated by the movement of the payload. It is indeed possible that in the case of high precision requests, feedback control approaches such as PID, Lead-Lag or LQR are not able to satisfy by themselves severe specifications. Hence different schemes, operating selectively on the stage direct disturbances, are required.

In this section an off-line feedforward scheme allowing to isolate the machine from the action of payload direct disturbance in operating condition is proposed. The scheme is not classical, i.e. the command is not generated on-line but it is computed in advance on the basis of the data response to the direct disturbance and the transfer function between the control command and the controlled output. As illustrated in Figure 3, the action of feedforward control is superimposed to the one of the Lead-Lag feedback control and acts exclusively on the disturbance acting from the payload.

The technique is based on the complete knowledge of the fixed pattern followed by the payload of the machine during operations. Since also the operation timing is known, it is possible to compute in advance a feedforward command, so as to be able to suppress the effects of the direct disturbance that are generated by the payload movements, and that cannot be measured. These commands are stored in the electronic control unit and are summed to the feedback control action at the appropriate time.

The model used to design the control law is the four degrees of freedom model exposed in Section 4.1. Being the XZ-plane and YZ-plane symmetric, just the latter is considered in the design phases.

The controlled output is the velocity measured on the stage $v_{s}(s)$ and it can be considered as the sum of two contributions: the effect of the direct disturbance on the output $v_{D s}(s)$ and the effect of the feedforward action on the output $v_{F F S}(s)$. Then the total response is:

$$
v_{s}(s)=v_{D s}(s)+v_{F F s}(s)=v_{D s}(s)+h(s) u_{F F}(s)
$$

where $h(s)$ is the transfer function between the control command $u_{F F}(s)$ to the controlled output $v_{F F}(s)$.

The control signal is:

$$
u_{F F}(s)=-h(s)^{-1} v_{D s}(s)
$$


Since the operation pattern and timing are known (Figure 23 (a)), the transfer function $h(s)$ can be obtained by using an FFT analyzer, the command signal $u_{F F}(s)$ (Figure 23 (b)) can be computed offline, stored in the control unit and applied to the system at the proper time when the payload is moving.

It is worthy to notice that the inversion of $h(s)$ leads to a non-causal function with a numbers of zeros equal or higher than the number of poles. This issue is overcome by adding the required number of poles at a frequency sufficiently high (more than $100 \mathrm{~Hz}$ ), in order to make the feedforward filter proper and fit to be used in the control scheme.

Bode diagram of $h(s)$ is reported in Figure 22 (feedback control is on, vibrations coming from the ground are damped).

Figure 23 (c) shows that the proposed technique is effective and allows to isolate the machine from the direct disturbance generated by the payload operations. The excitation signal reproduces a standard laser cut periodic profile.

The coupling of this action with the feedback control system permits to obtain a full vibration damping and active isolation from external disturbance coming from the ground and direct disturbance coming from the stage.

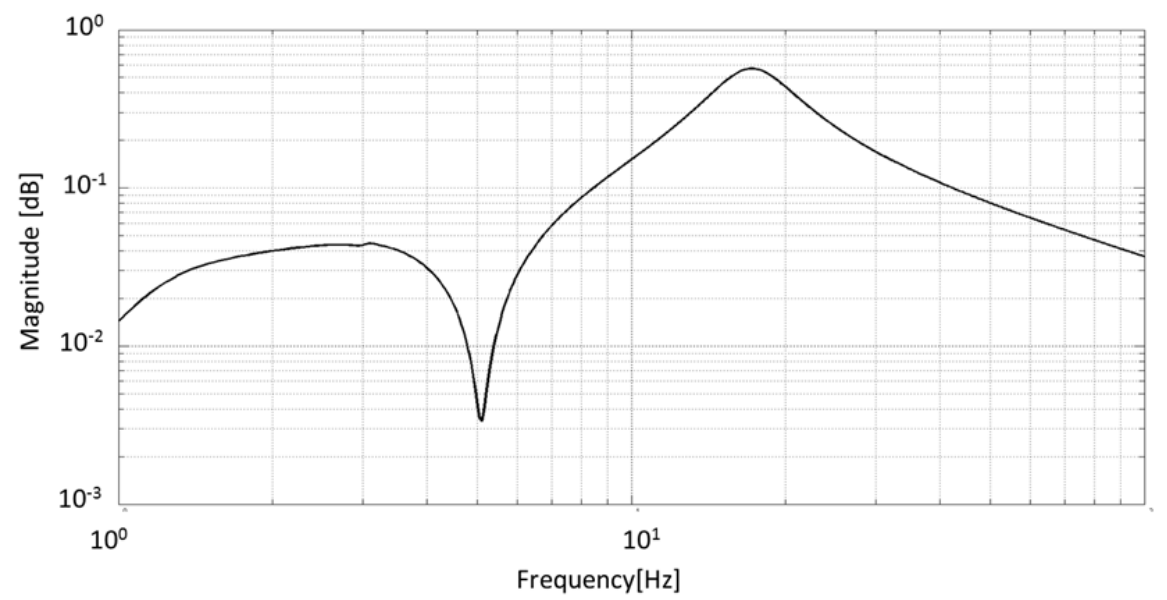

Figure 22. Control command to controlled output stage velocity transfer function (h(s)) Bode diagram. 

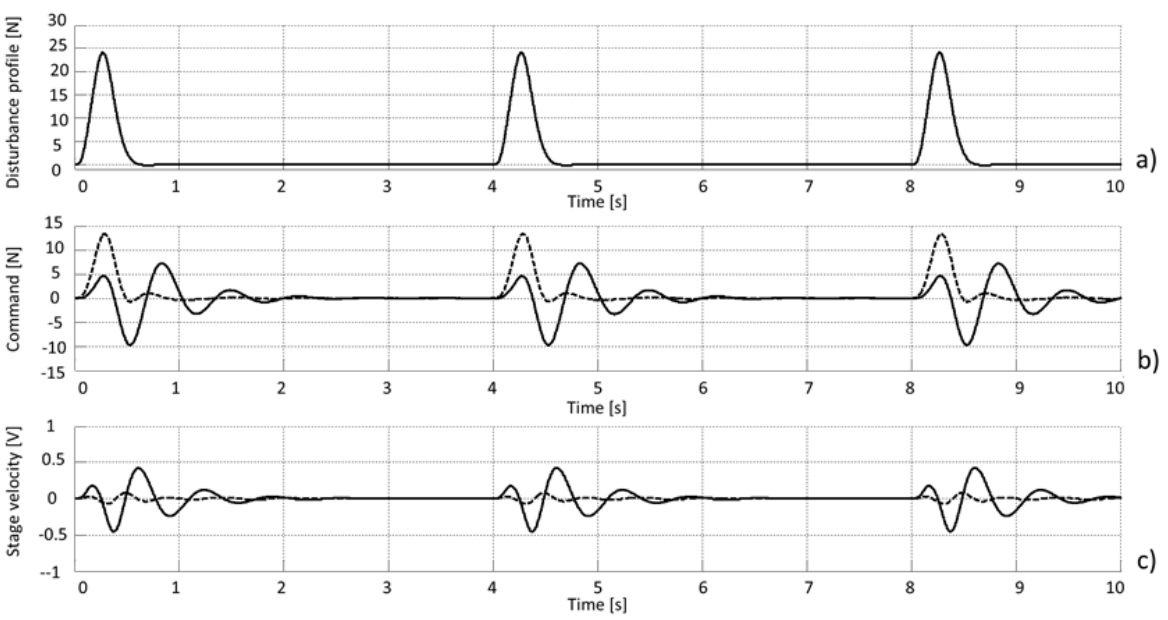

Figure 23. a) Feedforward control: disturbance profile; b) Control signal. Solid line: feedforward off, dashed line: feedforward on; c) Controlled output: stage velocity. Solid line: feedforward off, dashed line: feedforward on.

\subsection{Modal control}

The third and last control technique proposed in this chapter is a modal approach to perform a feedback control scheme. This strategy is similar in performance to the Lead-Lag strategy illustrated in Section 5.1, but it simplifies the control design procedure once it gives a direct feeling on actuators action on machine modes.

The method is based on the scheme reported in Figure 24. The goal of the technique is to decouple the rotational and translational motion modes of the machine to direct the action of the controller selectively on the dynamic of interest.

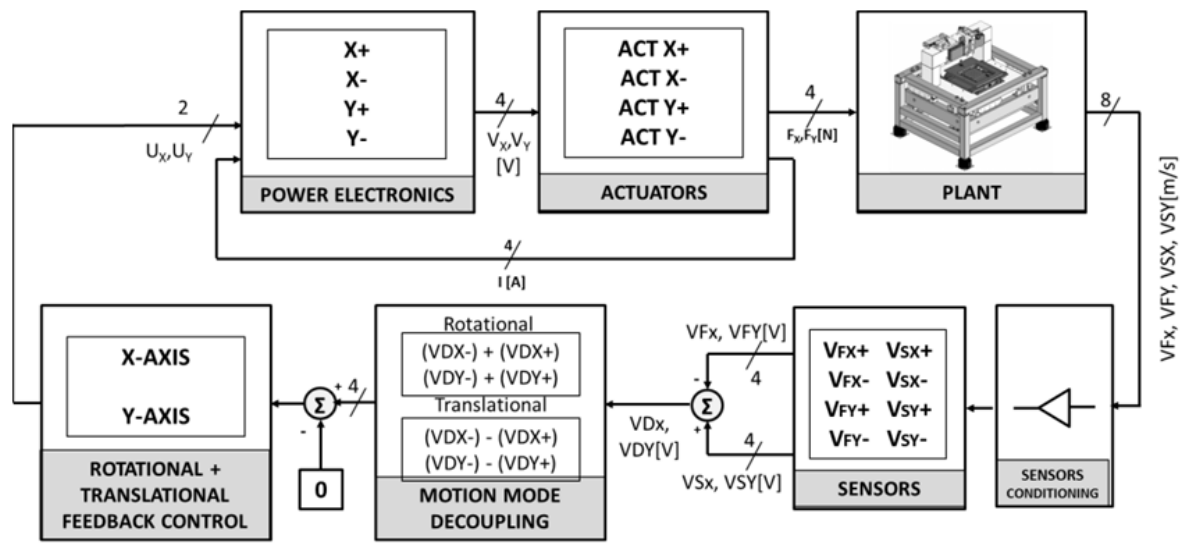

Figure 24. Modal control overall scheme. 
The eight geophones measurements on stage and frame are elaborated to obtain four velocity differences:

$$
\begin{aligned}
& V_{D X+}=V_{\text {SensSX+ }}-V_{\text {SensFX+ }} \\
& V_{D X-}=V_{\text {SensSX- }}-V_{\text {SensFX- }} \\
& V_{D Y+}=V_{\text {SensSY+ }}-V_{\text {SensFY+ }} \\
& V_{D Y_{-}}=V_{\text {SensSY- }}-V_{\text {SensFY- }}
\end{aligned}
$$

These values are then summed and subtracted in order to obtain the motion mode uncoupling.

Rotational mode:

$$
\begin{aligned}
& V_{R X}=V_{D X+}+V_{D X-} \\
& V_{R Y}=V_{D Y+}+V_{D Y-}
\end{aligned}
$$

Translational mode

$$
\begin{aligned}
& V_{T X}=V_{D X+}-V_{D X-} \\
& V_{T Y}=V_{D Y+}-V_{D Y-}
\end{aligned}
$$

The control dynamic is the same of Lead-Lag approach, the difference consisting in the error fed to the controller. The poles of the system in open and closed loop are reported in Table 4.
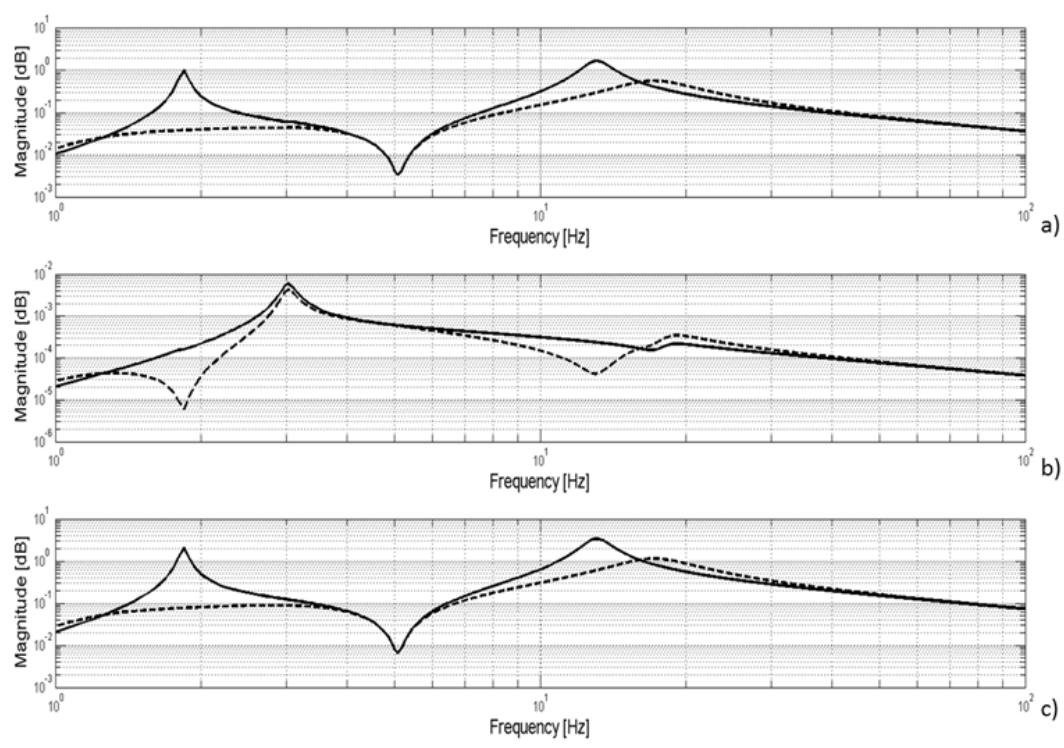

Figure 25. Modal control. a) Control command to stage-frame velocities difference transfer function. $b$ ) Control command to translational dynamics transfer function. c) Control command to rotational dynamics transfer function. Solid line: open loop. Dashed line: closed loop. 
Figure 25 shows the motion modes uncoupling and system behaviour in open and closed loop. Figure 25.a illustrates control command to stage-frame velocities difference transfer function where translational and rotational modes are coupled. Figure 25.b and Figure 25.c report the translational $\left(V_{T X, T Y}\right)$ and rotational $\left(V_{R X, R Y}\right)$ dynamics respectively. It is worthy to notice that the influence of rotational dynamics is dominant, being its response amplitude higher than translational one. Due to this consideration it can be easily explained the low action of the feedback control on the translational dynamics (b)) is compared to the rotational one (c)).

\section{Conclusions}

In this chapter the design of three different control techniques for vibration damping and active isolation for high precision laser cutting machines has been illustrated. After an overview on the main actuation technologies in this field the work explains the advantages of electromechanical actuators and focuses on the mechatronics approach of the machine subsystem design. For controller implementation, two different models (four and six degrees of freedom) have been developed. The considered controllers are:

a. Feedback control with a Lead-Lag approach;

b. Off-line Feedforward scheme;

c. Modal control.

Experimental and simulation results used to check the effectiveness of the modeling approach and of the three proposed control techniques.

\section{Author details}

Andrea Tonoli ${ }^{1,3}$, Angelo Bonfitto ${ }^{3}$, Marcello Chiaberge ${ }^{2,3}$,

Mario Silvagni ${ }^{1,3}$, Lester D. Suarez ${ }^{3}$ and Enrico Zenerino ${ }^{1,3}$

1Department of Mechanical and Aerospace Engineering/ Politecnico di Torino, Italy

2Department of Electronics and Telecommunications/ Politecnico di Torino, Italy

3Mechatronics Lab/Politecnico di Torino, Italy

\section{References}

Anderson E., Leo D.J., Holcomn M.D, (1996). UltraQuiet platform for active vibration isolation, Proceedings Smart Structures and Integrated Systems 2717, San Diego, CA, pp. 436-451.

Beadle B.M., Hurlebaus S., Gaul L., Stöbener U. (2005). Active control strategies for vibration isolation, in Proc. IUTAM Symp. Vibration Control of Nonlinear Mechanisms and Structures 2005, Munich, Germany, pp. 91-100.

Crede, C. (1951). Vibration and shock isolation, John Wiley and Sons Inc., New York, USA

Elliott S.J. (2001). Signal processing for active control, Academic Press.

Hauge, G.S. Campbell, M.E.. (2004). Sensors and control of a space-based six-axis vibration isolation system. Journal of Sound and Vibration 269. Pp 913-931. 
Huan, S. L., \& Pater, A. R. (1985). Analysis and prediction of geophone performance parameters. Geophysics, 50(8), 1221:1228.

Hyde T.T, (1997) An experimental study of active isolation. Proceedings of the 38th AIAA/ASME/ASCE/AHS/ASC, Structures, Structural Dynamics and Materials Conference, Orlando, FL, pp. 1763-1773.

Kuo S.M., Morgan D.R., (1996). Active noise control systems: algorithms and DSP implementations, John Wiley \& Sons.

Preumont, A. (2002). Vibration control of Active Structures. Kluwer Academic Publishers, $2^{\text {nd }}$ Edition, Netherlands

Riedesel, M., Moore, R. D., \& Orcutt, J. A. 1990. Limits of Sensitivity of Seismometers with Velocity Transducers and Electronic Amplifiers. Bulletin of the Seismological Society of America, 80(6), 1725:1752.

Rivin, E. I. (1979). Principles and criteria of vibration isolation of machinery. Trans. of the ASME, Journal of Mechanical Engineering, 101:682-692.

Sullivan J., Rahman Z., Cobb R., Spanos J. (1997). Closed-loop performance of a vibration isolation and suppression system, Proceedings of the American Control Conference, Vol. 6, Albuquerque, NM, pp. 3974-3978.

Thayer, D., Vagners, J., von Flotow, A., Hardham, C. and Scribner, K., (1998). "Six Axis Vibration Isolation Using Soft Actuators and Multiple Sensors," Proceedings of the 21st Annual AAS Guidance and Control Conference, Feb 1998.

Thomson, W. (1971). Theory of vibration with applications. George Allen and Unwin.

Tonoli A., Bonfitto A., Silvagni M., Suarez L.D., Zenerino E. (2011) Active Isolation and Damping of Vibrations for High Precision Laser Cutting Machine. In: Vibration Control/Beltrán-Carbajal F. InTech, Rijeka. ISBN 9789533074337

Watters B.G., Coleman R.B., Duckworth G.L., Berkman E.F. (1988). A perspective on active machinery isolation, Proceedings of the 27th Conference on Design and Control, Vol. 3, Austin, TX, pp. 2033-2038. 